\title{
Spinal Versus General Anesthesia for the Outpatient Unicompartmental Knee Arthroplasty: A Randomized Controlled Trial
}

\section{Chang Chen}

Southwest Medical University

Yiran Yin

Southwest Medical University

Huan Xu

Southwest Medical University

Ge Chen ( $\nabla 1707142602 @ q q . c o m)$

Department of Orthopedics, Affiliated Hospital of Southwest Medical University. Address:Luzhou, 646000,Sichuan Province, China

\section{Research article}

Keywords: unicompartmental knee arthroplasty(UKA), outpatient surgery, osteoarthritis, Spinal anaesthesia, general anaesthesia, short-term efficacy

Posted Date: December 2nd, 2020

DOI: https://doi.org/10.21203/rs.3.rs-118248/v1

License: (c) (1) This work is licensed under a Creative Commons Attribution 4.0 International License. Read Full License 


\section{Abstract}

Purpose: To compare the early clinical outcomes between anesthetic techniques in outpatient unicompartmental knee arthroplasty (UKA) and investigate factors responsible for delayed discharge.

Method: A randomized controlled trial was conducted on 64 consecutive and unselected patients who underwent primary, medial UKA and randomly divided into two groups:32 patients with spinal anaesthesia(Group A) and 32 with general anaesthesia(Group B). There were no significant differences in gender, age, body mass index (BMI), the hospital for Special Surgery knee score (HSS), and American Society of Anesthesiology(ASA) class between the two groups $(P>0.05)$.

Results囚Totally 64 patients were involved, including 20 males and 42 females, with an average age of $60.4 \pm 3.8$ year-old. The length of stay (LOS) and total expense were significantly less in group A than those in group $B(P<0.05)$. There was no significant difference between two groups on the discharge day and the reasons for not discharge $(P>0.05)$. Lack of mobilization (84\%), pain $(23 \%)$, and PONV $(17 \%)$ were main reasons for patients not meeting the discharge criteria on DOS (Table 2). Patients with spinal anaesthesia underwent a longer surgery time than those with GA. $(P<0.05)$; Patients in the Group $A$ had a significantly earlier oral fluid intake and walking unaided $(P<0.05)$; Pain occurred significantly earlier in Group $B(P<0.05)$.

Conclusion: In patients who underwent outpatient UKA, spinal anaesthesia is significantly superior to general anaesthesia with shorter discharge time and lower cost. With strict patient selection and safe anaesthesia, outpatient UKA could attain a good clinical outcome with both anaesthesia techniques.

\section{Background}

Unicompartmental knee arthroplasty (UKA) is a safe and effective intervention for end-stage knee arthritis confined to the medial compartment[1][2]. Compared with total knee arthroplasty (TKA), preservation of cruciate ligaments and less bone loss allowed UKA to attain a earlier recovery, less perioperative complications, and a better range of motion(ROM) of knee[3][4].

In recent years, the improvement of surgical techniques and the development of Enhanced Recovery after Surgery (ERAS) management allowed UKA to be performed in a same-day, outpatient setting[5][6]. Outpatient UKA has been widely performed and associated with decreased length of stay compared to inpatient procedure without increased risk of major complications[7][8]. Outpatient joint arthroplasty could effectively reduce the LOS, and thus, minimize the incidence of nosocomial infection[9]. Few studies investigated how to carry out outpatient UKA more safely and effectively [10]. Multiple aspects of the perioperative care which might affect outcomes, including type of anesthesia, preoperative medications, optimization of medical comorbidities, fluid management, postoperative analgesia and discharge criteria have not been fully studied[11]. Unsatisfactory pain management might lead to prolonged length of stay(LOS) and increased cost, associated with decreased patient satisfaction[12]. Whether types of anaesthesia have potential impact on perioperative outcomes of outpatient UKA 
remains controversial. Several studies suggested that SA reduces the rate of complications and healthcare resource utilization[13][14]. But there were few studies specifically examined those patients who would be eligible to be outpatient UKA candidates.

Thus, we conducted a randomized controlled trial in a single institution. The purpose of this study was to compare the clinical efficacy and early complications between spinal anaesthesia(SA) and general anaesthesia(GA) in patients who underwent outpatient unicompartmental knee replacement.

\section{Methods}

The inclusion criteria were as follows: (1) end-stage medial compartment osteoarthritis which was was clinically and radiographically diagnosis and met the surgical indications for unicompartmental knee arthroplasty (UKA); (2) American Society of Anesthesiology(ASA) classes 1 to 2, or class 3 but assessed could tolerate UKA; (3) those have a responsible caregiver and can stay with them for at least 4 days after operation; (4) patients and their caregiver can adequately understand the process of outpatient operation and post-operative analgesia regimen; (5) adequate home environment for postoperative recovery. Exclusion criteria included: (1) rheumatoid arthritis or suppurative arthritis;; (2)patients with systemic diseases and can not tolerate major surgical operation (ASA 3-5); (3) opioid consumption (4)patients with contraindications of spinal anesthesia or femoral nerve block (FNB); (5)refused to participate in this study or not willing to finish regular follow-up.

After approved by the Ethics Committee of our institution (KY2020124) , a randomized controlled trial was conducted on the basis of pre-and postoperative outpatient clinical charts. From October 2017 to June 2018, 64 consecutive and unselected patients who underwent primary, medial UKA in our institution were involved in our study. All patients were operated with cementless mobile bearing Oxford $₫$ UKA system with a minimal invasive technique as described by the manufacturer. A single senior surgeon performed more than 200 primary UKAs, in which the outpatient procedures was employed in $26.7 \%$ of cases. After assessed by the senior surgeon, patients who met the inclusion criteria for day-time UKA operation could be involved in our study. 64 patients were equally divided into two groups using random number method:32 patients with spinal anaesthesia(group A) and 32 with general anaesthesia(group B). All patients participating in the trial gave written informed consent.

\section{Patients and procedures}

Patients who met the inclusion criteria could be scheduled for the operation. Written informed consent was obtained before inclusion in the study.

- Two weeks preoperatively: Comprehensive educational material would be provided by our surgeon, and the degree of preoperative pain was evaluated by visual analog score (VAS). Patients with VAS score $\geq 4$ either at rest or in motion were prescribed with oral analgesia: NSAIDs (celecoxib), pregabalin, or buprenorphine for external use. 
- One week preoperatively: Senior surgeon and anaesthesiologist should reassess and confirm if patient met the inclusion/exclusion criteria. Patients were contacted by the protocol nursing, who would provide the introductions about the surgical procedure, anaesthesia techniques, risks and recovery procedures. Further preoperative education, including strict prohibition of smoking and alcohol, ankle pump exercise and quadriceps muscle contraction training, schedule of rehabilitation plan and daily goals of functional exercise after operation should also be informed to help them establish a reasonable psychological expectation, and make them fully understand and participate in recovery process.

- Three days preoperatively: Chest X-ray, electrocardiogram and other auxiliary examinations should be implemented. Patients with anemia (HB: female $<110 \mathrm{~g} / \mathrm{L}$, male $<120 \mathrm{~g} / \mathrm{L}$ ) or hypoalbuminemia (< $30 \mathrm{~g} / \mathrm{L}$ ) should be delayed until their HB/albumin returned to normal level; For patients with hypertension and diabetes mellitus, the blood glucose and blood pressure should be adjusted to the normal range, then the operation could be scheduled again.

- Day of operation: When arrived at surgical department, all patients were randomized arranged into one of two groups(Group A or Group B). After confirmed no absolute contraindication, related documents could be signed. Fasting solid food 6 hours before induction; 2 hours before induction, $600 \sim 800 \mathrm{ml}$ high concentration carbohydrate were given orally. Prophylactic antibiotic and a single dose $(1 \mathrm{~g})$ of tranexamic acid was given intravenously 30 minutes before skin incision.

Types of anaesthesia

The senior surgeon performed THA on all patients by using the same surgical technique and perioperative protocol for both groups. And all anesthetic procedures(SA and GA) were under the management of an experienced anesthetist. All patients were given femoral nerve block (FNB) preoperatively.

\section{Spinal anaesthesia}

Under aseptic conditions, spinal anaesthesia was performed in a midline approach. The anaesthetist identified the L3 to L4 interspace in the lateral decubitus position. After local infiltration anaesthesia(LIA), the subarachnoid space was sought. Free-flowing cerebrospinal fluid(CSF) and no reflux of blood indicated correct needle position, then $2 \mathrm{~mL} 0.5 \%$ hyperbaric bupivacaine was injected in the absence of paraesthesia. Blood pressure and heart rate should be carefully observed. After induction patients were immediately transferred to the operating room and positioned. Optionally, by request, patients were given propofol until a mild level of sedation was achieved (Richmond Agitation Sedation Scale 1 to 2). Sensory block and motor block were evaluated 3 min after injection. Sensory block was tested using a toothpick to elicit pinprick starting caudally and moving cephalad.

\section{General anaesthesia}

After pre-oxygenation, sufentanil (0.2 0.5mg/ $\mathrm{kg}$ ) and propofol ( 2 to $3 \mathrm{mg} / \mathrm{kg}$ ) were intravenously injected. Laryngeal mask (LM) was provided according to body weight. For difficult or impossible 
ventilation that could not be resolved by replacement or re-insertion of the $L M$, an endotracheal tube would be provided with an inner diameter of $7.0 \mathrm{~mm}$ for women and $7.5 \mathrm{~mm}$ for men. The nondepolarising neuromuscular blocking agent atracurium was administered for orotracheal intubation. Ventilation was managed by pressure-controlled mode at a frequency to achieve an end tidal $\mathrm{PCO}_{2}$ of 35 $\sim 40 \mathrm{mmHg}$. Anaesthesia was maintained by continuous propofol infusion through a perfusor pump, with the infusion rate set $8 \sim 12 \mathrm{mg} \mathrm{kg} /$ body weight/h, and could be adjusted according to the individual condition. To ensure prompt recovery, the infusion of propofol was stopped 5 min before completion of skin closure. If necessary, the anaesthetist would administer repetitive sufentanil doses according to clinical need. Indicated by the Apfel score, patients at risk of PONV received granisetron $(1 \mathrm{mg})$ and dexamethasone $(4 \mathrm{mg})$ as prophylaxis.

Surgical technique

After a satisfactory anaesthesia, all patients were put in hanging leg $(\mathrm{HL})$ position, then an iodised incision drape (loban $3 \mathrm{M} \AA$ ) was applied after conventional draping. All the surgical incisions were performed by minimally invasive surgical incision near the patella. Oxford $\otimes$ UKA prostheses were utilized in all patients. The senior doctor with a fixed surgical team performed operation according to the Oxford III UKA system operation manufacturer. Before skin suture, all patients received local infiltration analgesia (LIA) with $20 \mathrm{ml}$ "cocktail" (ropivacaine $200 \mathrm{mg}$, epinephrine $5 \mathrm{mg}$, triamcinolone acetonide $5 \mathrm{mg}$ ) injected in the posterior capsule, periarticular tissues, and subcutaneous tissue. No intra-articular suction drain or urinary catheter was placed in all patients. The fluoroscopy with a C-arm X-ray machine should be conducted to ascertain that the prostheses were satisfactory. Once the procedure was finished, all patients were immediately transferred into the postanesthesia care unit(PACU). After pain and PONV were controlled, patients were orally provided with $200 \mathrm{ml}$ high concentration carbohydrate and sent back to the ward after active bleeding disappeared.

Postoperative management

All patients were treated by multimodal analgesia once sent back to ward, in combination with PCIA (Patient control intravenous anesthesia) pump. 2 hours after surgery, patients were orally given celecoxib $(200 \mathrm{mg} / 12 \mathrm{~h})$, Tylenol $(650 \mathrm{mg} / 8 \mathrm{~h})$ and gabapentin $(300 \mathrm{mg} / 8 \mathrm{~h})$. If the pain symptoms were still obvious (VAS score $\geq 5$, and the pain affected postoperative functional exercise), tramadol and buprenorphine were given additionally. $1 \mathrm{~h}, 3 \mathrm{~h}$ and $24 \mathrm{~h}$ postoperatively, single dose of tranexamic acid $(1 \mathrm{~g})$ was given separately.

Postoperative functional exercise was initiated and evaluated by the therapists in day-time operation team. After the effect of anesthesia subsided, the functional exercise should be started, including CPM, ankle pump, knee flexion and extension exercise. 2 hours after operation, all patients were required to sit and stand up. Then with the walking aid, they were required to try to walk independently with adjustable lower limb brace. The therapists ensured that all patients can walk safely and, meanwhile, assessed their 
gait and pain level. 4 hours after the operation, they walked on the floor again and tried to walk up and down stairs independently.

The discharge criteria were as follows: 1) Mobilizes in and out of bed independently; 2) walk $30 \mathrm{~m}$ independently; 3) walk up and down stairs independently; 4) obvious relief of pain; 5) has minimal or no wound wound exudation; 6) $\mathrm{HB} \geq 90$; 7) patient feel comfortable and confident to go home.

Only patients met the discharge criteria can they be discharged. Anticoagulation with low molecular weight heparin was given every day from 12 hours after operation, and rivaroxaban was given orally after discharge until 3 weeks after operation;

All discharged patients were maintained contact via Wechat, a social networking application (Tencent Inc., China) and telephone. They were followed up by a doctor on regular basis.

The primary outcome measures included the length of stay (LOS), the total hospitalization cost. Early post-operative complications and reasons for delayed discharge were recorded. And the operation time, intraoperative blood loss and VAS at the time of discharge were recorded. Patients were followed up regularly $1,3,6,12$ month(s) and then annually after the operation, to record the complications, including periprosthetic infection, thromboembolic event, prosthesis dislocation and aseptic loosening. And the hospital for Special Surgery knee score (HSS) and Oxford knee score (OKS) were used to assess the knee function.

Study size and statistical methods

The sample size was calculated according to the primary outcome (the length of stay) using the SAS software package (version 9.0, SAS Institute Inc., lowa, IA, USA). We presumed that a difference of $2 \mathrm{~h}$ between the two groups would be clinically important. Assuming a SD of $4 \mathrm{~h}$, a $5 \%$ significance level and a $90 \%$ power using a two-sided test, we obtained a total sample size of $n=22$ for each group. As we were not able to ensure that these data would be normally distributed, we enhanced the sample size to $n=32$ patients per group.

SPSS 22.0 statistical software was employed for statistical analysis. Measurement data were expressed as mean \pm standard deviation (SD). Repeated analysis of variance was used for comparison between time points before and after surgery in the groups. Paired t-test was used for pairwise comparison. Independent sample t test was utilized for comparison between groups. Comparison of count data between groups was made by using $\chi 2$ test. Inspection level was set at $\mathrm{a}=0.05$.

Results

A total of 64 patients were involved, consisting of 20 males and 42 females, with an average age of 60.4 \pm 3.8 year-old at the time of surgery. The demographics are shown in Table 1. There existed no significant differences in demographics between the two groups, including sex $(P=0.59)$, age $(P=0.96), \mathrm{BMI}(P=$ 0.83), ASA ( $P=0.74)$, and preoperative HSS $(P=0.47)$. 
Tab.1 Comparison of Patient Demographics. 0

\begin{tabular}{|lllllll|}
\hline Group & $\mathrm{n}$ & $\begin{array}{l}\text { Sex } \\
\text { male/female }\end{array}$ & Age & BMI & ASA & Preoperative HHS \\
\cline { 1 - 2 } & 32 & $9 / 23$ & $60.1 \pm 6.3$ & $22.80 \pm 4.45$ & $2.0 \pm 0.4$ & $46.81 \pm 7.37$ \\
\hline B & 32 & $11 / 21$ & $60.2 \pm 5.5$ & $23.03 \pm 5.52$ & $1.9 \pm 0.3$ & $48.44 \pm 4.92$ \\
& & & & & & $t=-1.009$ \\
Statistic & & & $t=-0.052$ & $t=-0.213$ & $t=0.330$ & $P=0.321$ \\
& & $P=0.590$ & $P=0.959$ & $P=0.833$ & $P=0.751$ & \\
& & & & & & \\
\hline
\end{tabular}

The length of stay(LOS) and total cost were significantly less in group $A$ than in group $B(P<0.05)$. The patients in group A were followed up for 6-16 months (mean time: 9.7 months) and 6-18 months (mean time: 9.3 months) in group B. In terms of postoperative satisfaction, the numeric rating scales (NRS) of group A was (8.87 \pm 0.83$)$, which was slightly higher than that of group B $(8.59 \pm 0.84)$, and the difference was not statistically significant $(P>0.05)$ (see Table 2 ).

Tab.2 Comparison of clinical indexes when discharge between 2 groups 0

\begin{tabular}{|c|c|c|c|c|c|c|}
\hline Group & $\mathrm{n}$ & LOS(h) & Expense囚¥囚 & NRS & 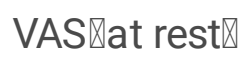 & VAS『in motion $\rrbracket$ \\
\hline$A$ & 32 & $17.7 \pm 3.9$ & $40356.5 \pm 652.8$ & $8.87 \pm 0.83$ & $2.06 \pm 0.31$ & $3.15 \pm 0.48$ \\
\hline \multirow[t]{2}{*}{ B } & 32 & $21.4 \pm 6.4$ & $44131.8 \pm 191.7$ & $8.59 \pm 0.84$ & $2.13 \pm 0.49$ & $3.25 \pm 0.58$ \\
\hline & & $t=-0.052$ & $t=-0.052$ & $t=-0.213$ & $t=0.330$ & $t=-1.009$ \\
\hline Statistic & & $P=0.590$ & $P=0.959$ & $P=0.833$ & $P=0.751$ & $P=0.321$ \\
\hline
\end{tabular}

Among all patients, 51 patients (80\%) were not discharged on the day of operation (DOS), with 53 patients(83\%) discharged on POD-1, and 95\% on POD-2. In group A, 24 patients were not discharged on the day of operation (DOS), with 27 patients discharged on POD-1, and 30 on POD 2; In group B, 27 patients were not discharged on the day of operation (DOS), with 26 patients discharged on POD-1, and 31 on POD 2. There was no significant difference between two groups on the discharge day ( $P>0.05)$. Lack of mobilization (84\%), pain (23\%), and PONV (17\%) were the main reasons for patients not meeting 
the discharge criteria on DOS (Table 3). There was no significant difference between two groups on the reasons for not discharge on DOS $(P>0.05)$.

Tab.3 count and percentage of total amount of patients still hospitalized on day of surgery (DOS), postopertive day 1 (POD 1) and postopertive day 2 (POD 2)

\begin{tabular}{|llll|}
\hline & \multicolumn{3}{l}{ Not discharged on } \\
\cline { 2 - 4 } group & DOS & POD 1 & POD 2 \\
\cline { 2 - 4 } & $\mathrm{N}=51$ & $\mathrm{~N}=11$ & $\mathrm{~N}=3$ \\
$\mathrm{~S}$ & 24 & 5 & 2 \\
$\mathrm{G}$ & 27 & 6 & 1 \\
\hline X2 & 1.463 & 0.110 & 0.350 \\
$\mathrm{P}$ & 0.226 & 0.704 & 1.000 \\
\hline
\end{tabular}

Patients with spinal anaesthesia underwent a longer surgery time than those with $G A$. $(P<0.05)$; And the blood loss in two groups had no significant difference $(P=0.727)$. Meanwhile, Patients in the Group A had a significantly earlier oral fluid intake and walking unaided $(P<0.05)$; Pain occurred significantly earlier in the Group $B(P<0.05$; Table 4$)$.

Tab.4 Duration of procedures and recovery times from start of intrathecal injection 0

\begin{tabular}{|llll|}
\hline Group & A & B & P value \\
\hline Operation time (min) & $70.8 \pm 7.1$ & $60.1 \pm 9.1$ & 0.000 \\
\hline Blood loss (ml) & $121.3 \pm 15.8$ & $122.3 \pm 19.1$ & 0.727 \\
\hline Occurrence of pain (min) & $108.8 \pm 10.0$ & $71.8 \pm 8.4$ & 0.000 \\
\hline Food intake (min) & $55.4 \pm 4.5$ & $91.8 \pm 5.3$ & 0.000 \\
\hline Walking unaided (min) & $119.7 \pm 15.5$ & $139.3 \pm 13.9$ & 0.000 \\
\hline
\end{tabular}

Time spans marked with an $\mathrm{M}$ are measured from the end of induction; median (range) are given. 
Figure 1 showed the comparison of HSS score and OKS score between the two groups preoperatively and post-operatively: the HSS score and OKS score of both groups were significantly improved at 1, 3 and 6 months after operation, and the differences between two groups were not statistically significant $(P>$ 0.05).

There was no significant difference in VAS scores between the two groups at the time of discharge $(P>$ 0.05), suggesting that while the LOS was significantly reduced, the pain symptoms could still be well controlled by both types of analgesia. At latest follow-up, no periprosthetic infection, aseptic loosening of prosthesis, deep vein thrombosis (DVT), pulmonary infection and other complications were found in all patients.( see Fig.2)

\section{Discussion}

The purpose of this randomized controlled trial was to compare the early clinical outcomes between anesthetic techniques in outpatient unicompartmental knee arthroplasty (UKA) and investigate factors responsible for delayed discharged. The results of our study showed that both spinal and general anesthesia can attain satisfactory short-term outcomes in outpatient UKA based on ERAS pathway and multimodal analgesia. Meanwhile, spinal anesthesia might be a more cost-effective than general anesthesia, which could reduce the LOS as well.

Compared to total knee arthroplasty (TKA), unicompartmental knee arthroplasty (UKA) is associated with an earlier recovery, less postoperative pain, lower morbidity and mortality, and a greater "feel" of a normal knee, which made UKA a better surgical option for patients with end-stage medial compartment osteoarthritis[15][16]. Because of not increasing postoperative complications and risk of re-admission, outpatient joint arthroplasty have been widely performed in recent years[17]. A shorter LOS could effectively save the cost of hospitalization and reduce the risk of nosocomial infection [18]. Current studies have confirmed the safety and efficacy of outpatient UKA [7][9][13]. Meanwhile, excellent anesthesia and intraoperative monitoring were necessary for ensuring the safety and efficacy of the operation. Though both spinal and general anaesthesia were appropriate techniques for outpatient UKA, it remained controversial which type of anaesthesia would attain a better clinical outcome.

A randomised controlled study conducted by Gebhardt et al [19] indicated that in patients of outpatient knee surgery, spinal anaesthesia can lead to a significantly earlier discharge and reduce total cost compared with general anaesthesia. A large national sample study by Memtsoudis et al [20] also showed that the utilization of neuraxial versus general anesthesia for primary joint arthroplasty was associated with superior perioperative outcomes. In our study, compare with GA, patients with SA did have a short mean LOS and lower cost of hospitalization. And both types of anaesthesia did not increase the incidence of post-operative complications. As for 30-day mortality, all patients in our study did not suffer any major post-operative complications at the latest follow-up.

Limited evidence suggested that SA could reduce total blood loss. A study of Perlas et al [21] suggested that a strong association between spinal anesthesia and lower 30-day mortality, as well as a less likely to 
experience major blood loss after elective joint replacement surgery. Poeran et al [22] provided incremental evidence of the potential effectiveness and safety of tranexamic acid in patients requiring orthopedic surgery, which indicated that tranexamic acid can effectively reduce the need for blood transfusions while not increasing the risk of complications, including thromboembolic events and renal failure. We also believed that the application of antifibrinolytics might effectively eliminate the differences. In our study, tranexamic acid were routinely used before and after the surgical procedure. And the result showed no significant difference between two groups in term of total blood loss, with routine use of anticoagulants. And at latest follow-up, all patients did not suffer venous thromboembolic events (VTEs), which also indicated the safety of tranexamic acid.

In this study, a total of 13 patients (20\%) completed DOS discharge, which was slightly lower when compared with most of current studies[9][10][13]. This may because of the criteria for discharge might be relatively conservative considering the safety of patients and inadequate experience of outpatient joint arthroplasty in our institution. Among all 51 patients who could not be discharged on DOS, lack of mobilization (84\%), pain (23\%), and PONV (17\%) were the main reasons for delayed discharge. A randomized controlled trial by Hoorntje et al [23] suggested that patients who lack or afraid of doing selfdirected exercise should not discharge on DOS. In our study, 43 patients were not discharged on the DOS due to the lack of mobilization, and the incidence in group $A(24 / 32,75.0 \%)$ was slightly higher than those in group $B(19 / 32,59.4 \%)$ without statistically significant difference $(\chi 2=0.741, P=0.245)$. And after recovery guidance by our therapists and application of CPM and other rehabilitation devices, most of them (37 cases) were successfully discharged in POD 1.

In this study, a total of 12 patients could not be discharged from hospital on DOS because of obvious pain, and the overall incidence was not significantly between group $A(7 / 32,21.9 \%)$ and group $B(5 / 32$, $15.6 \%)(\chi 2=0.355, P=0.384)$. We believed that while both anesthetic techniques had no difference in postoperative pain management, attention should be paid to achieve a good pain control for safe discharge of day-time surgery. Many studies have shown that adequate preoperative education, preemptive oral analgesia, nerve block and multimodal analgesia can effectively ensure the safety of day-time surgery [27]. A case-controlled study of hoorntje et al [25] suggested that effective pain management of outpatient UKA can reduce the anxiety and depression of patients during the perioperative period, enable patients to actively participate in rehabilitation training, achieve better clinical efficacy, and effectively improve patients' surgical satisfaction. On the other hand, a study of Kort et al [26] showed that the application of multimodal analgesia in UKA surgery can not only improve the perioperative analgesic efficiency, but also effectively reduce the use of opioids. In our study, at the time of discharge, the VAS score of both groups had no significant difference $(P>0.05)$. However, when considering first occurrence of pain after surgery, patients with general anaesthesia were significant earlier than those with spinal anaesthesia $(P>0.05)$, which might affect the efficacy of early function exercise. And patients in Group A also had a shorter LOS than Group A resulted from a better early function exercise. All patients were managed by multimodal analgesia, and only 5 patients ( 2 in Group A, 3 in Group B) were given tramadol and buprenorphine additionally due to obvious pain. Meanwhile, Turner et al. [27] indicated that compared with femoral nerve block(FNB), adductor canal block can 
achieve the same analgesic effect and has less impact on strength quadriceps, which is beneficial for patients to start early mobilization and recovery on DOS, and thus more suitable for outpatient UKA.

Poor quality of recovery and post-operative complications might increase the LOS and has an obvious impact on total cost. A cohort study by Baldawi et al [28] suggested that neuraxial anesthesia had reduced postoperative complications and LOS compared to patients who underwent general anesthesia. Chaturvedi et al [32] also indicated that in patients with a very high comorbidity burden, neuraxial anesthesia is a reasonable alternative to general anesthesia. However, it is uncertain whether those results are pertinent to outpatient total joint replacements considering that they were inpatient surgical procedures with total joint replacement. Our study specifically investigated the association between anesthetic techniques and early outcomes after outpatient UKA. In our study, all patients in two group did not suffer any major complication in their latest follow-up. Meanwhile,there was no significant difference in HSS and OKS score between two groups at 1, 3 and 6 months after operation, suggested that both anaesthesia techniques could attain satisfactory clinical outcomes.

There are still some limitations of this study. First, due to certain inherent features, the sample of our study may relatively be small. But the researchers, outcome assessors and patients were all blind to treatment groups, which might eliminated some biases. Second, all patients selected were mainly chosen by the surgeon, and this might result in some selection biases even if the senior surgeon did not know which group those patient were eventually divided into. Additionally, the sample size of the study was relatively small and both groups had very low complication rates, which rendered it hard to identify the association of the procedure with complications. These limitations could be avoided in future larger and long-term follow-up well-designed prospective randomized controlled studies.

\section{Conclusion}

Overall, our results suggest that, while both anaesthesia techniques for outpatient unicompartmental knee arthroplasty (UKA) are well tolerated and reliable, spinal anaesthesia is significantly superior to general anaesthesia with shorter time to discharge and lower cost of hospitalization. With strict patient selection and safe application of anaesthesia technique, outpatient UKA could attain a good clinical outcome.

\section{Abbreviations}

TKA: Total knee arthroplasty, UKA: Unicompartmental knee arthroplasty, ROM: range of motion, ERAS: Enhanced Recovery after Surgery, LOS: Length of stay, SA: Spinal anaesthesia, GA: general anaesthesia, ASA: American Society of Anesthesiology, FNB: femoral nerve block, HSS: the hospital for Special Surgery knee score, OKS: Oxford knee score, VAS: Visual analogue score, PACU: Postanesthesia care unit, PONV: Postoperative nausea and vomiting, DOS: Day of surgery, POD: Postoperative day, PCIA: Patient control intravenous anesthesia 


\section{Declarations}

Acknowledgements

Not applicable.

\section{Authors' contributions}

CC planned the research, analyzed the data and wrote the manuscript. CG and YY carried out the surgical procedure; $\mathrm{XH}$ and $\mathrm{YY}$ stored the clinical materials and were in charge of patients' follow-up; $\mathrm{CG}$ and $\mathrm{CC}$ planned the study, coordinated the whole study,. All the authors read and approved the manuscript.

\section{Funding}

This research is supported by the Cooperation program between Sichuan University and Luzhou municipal people's government (No. 2019cdlz-17).

\section{Availability of data and materials}

The datasets analyzed during the current study are available from the corresponding author on reasonable request.

\section{Ethics approval and consent to participate}

This study was approved by the Ethics Committee of The Affiliate Hospital of Southwest University.

\section{Consent for publication}

Informed consent was obtained from all individual participants included in the study.

\section{Competing interests}

The authors declare that they have no competing interests

\section{References}

1. Tapasvi S, Shekhar A, Patil S, et al.Limb position influences component orientation in Oxford mobile bearing unicompartmental knee arthroplasty: an experimental cadaveric study.[J].Bone Joint Res.20209(6):272-278

2. Hefny MH, Smith NA, Waite J, Cementless medial Oxford unicompartmental knee replacement. Fiveyear results from an independent series.[J].Knee.202027(4):1219-1227

3. Pongcharoen $\mathrm{B}$, Timjang $\mathrm{J}$, The outcomes of mobile bearing unicompartmental knee arthroplasty and total knee arthroplasty on anteromedial osteoarthritis of the knee in the same patient.[J].Arch Orthop Trauma Surg.2020(): 
4. Cheng J, Feng M, Cao G, et al.Patient outcomes in Anteromedial osteoarthritis patients over 80 years old undergoing Oxford Unicompartmental knee Arthroplasty in China.[J].BMC Musculoskelet Disord.202021(1):446

5. Mahure SA, Feng JE, Schwarzkopf RM, et al.Differences in Pain Opioid Use and Function Following Unicompartmental Knee Arthroplasty compared to Total Knee Arthroplasty.[J].J Arthroplasty.2020():

6. Nakasone CK, Combs D, Buchner B, et al.Day of surgery discharge success after implementation of a rapid discharge protocol following unilateral unicompartmental knee arthroplasty. [J].Knee.202027(3):1043-1048

7. Bosch LC, Bala A, Denduluri SK, et al.Reimbursement and Complications in Outpatient vs Inpatient Unicompartmental Arthroplasty.[J].J Arthroplasty.202035(6S):S86-S91

8. Kahlenberg CA, Richardson SS, Gruskay JA, et al.Trends in Utilization of Total and Unicompartmental Knee Arthroplasty in the United States.[J].J Knee Surg.2020():

9. Ford MC, Walters JD, Mulligan RP, et al.Safety and Cost-Effectiveness of Outpatient Unicompartmental Knee Arthroplasty in the Ambulatory Surgery Center: A Matched Cohort Study. [J].Orthop Clin North Am.202051(1):1-5

10. Schwenk ES, Johnson RL, Spinal versus general anesthesia for outpatient joint arthroplasty: can the evidence keep up with the patients?[J].Reg Anesth Pain Med.2020():

11. Memtsoudis SG, Cozowicz C, Bekeris J, et al.Anaesthetic care of patients undergoing primary hip and knee arthroplasty: consensus recommendations from the International Consensus on AnaesthesiaRelated Outcomes after Surgery group (ICAROS) based on a systematic review and meta-analysis. [J].Br J Anaesth.2019123(3):269-287

12. Li P, Kennedy J, Mohammad HR, et al.Acceptable outcomes with unicompartmental knee replacement and PCL deficiency are achievable: a case series of nine patients.[J].Knee Surg Sports Traumatol Arthrosc.2020():

13. Xu J, Cao JY, Chaggar GS, et al.Comparison of outpatient versus inpatient total hip and knee arthroplasty: A systematic review and meta-analysis of complications.[J].J Orthop.2020170:38-43

14. Johnson RL, Kopp SL, Burkle CM, et al.Neuraxial vs general anaesthesia for total hip and total knee arthroplasty: a systematic review of comparative-effectiveness research.[J]. $\mathrm{Br} \mathrm{J}$ Anaesth.2016116(2):163-76

15. Bosch LC, Bala A, Denduluri SK, et al.Reimbursement and Complications in Outpatient vs Inpatient Unicompartmental Arthroplasty.[J].J Arthroplasty.202035(6S):S86-S91

16. Cody JP, Pfefferle KJ, Ammeen DJ, et al.Is Outpatient Unicompartmental Knee Arthroplasty Safe to Perform at an Ambulatory Surgery Center? A Comparative Study of Early Post-Operative Complications.[J].J Arthroplasty.201833(3):673-676

17. Ruiz N, Buisson X, Filippi G, et al.Ambulatory unicompartmental knee arthroplasty: Short outcome of 50 first cases.[J].Orthop Traumatol Surg Res.2018104(7):961-966

18. Wyles CC, Vargas-Hernandez JS, Carlson SW, et al.Single-Dose Perioperative Antibiotics Do Not Increase the Risk of Surgical Site Infection in Unicompartmental Knee Arthroplasty.[J].J 
Arthroplasty.201934(7S):S327-S330

19. Gebhardt V, Zawierucha V, Sch?ffski O, et al.Spinal anaesthesia with chloroprocaine $1 \%$ versus total intravenous anaesthesia for outpatient knee arthroscopy: A randomised controlled trial.[J].Eur J Anaesthesiol.201835(10):774-781

20. Memtsoudis SG, Sun X, Chiu YL, et al.Perioperative comparative effectiveness of anesthetic technique in orthopedic patients.[J].Anesthesiology.:1046-58

21. Perlas A, Chan VW, Beattie S, Anesthesia Technique and Mortality after Total Hip or Knee Arthroplasty: A Retrospective Propensity Score-matched Cohort Study.

[J].Anesthesiology.2016125(4):724-31

22. Poeran J, Rasul R, Suzuki S, et al.Tranexamic acid use and postoperative outcomes in patients undergoing total hip or knee arthroplasty in the United States: retrospective analysis of effectiveness and safety.[J].BMJ.2014349():g4829

23. Hoorntje A, Waterval-Witjes S, Koenraadt KLM, et al.Goal Attainment Scaling Rehabilitation Improves Satisfaction with Work Activities for Younger Working Patients After Knee Arthroplasty: Results from the Randomized Controlled ACTION Trial.[J].J Bone Joint Surg Am.2020():

24. Fillingham YA, Darrith B, Lonner JH, et al.Formal Physical Therapy May Not Be Necessary After Unicompartmental Knee Arthroplasty: A Randomized Clinical Trial.[J].J Arthroplasty.201833(7S):S93S99.e3

25. Hoorntje A, Koenraadt KLM, Boevé MG, et al.Outpatient unicompartmental knee arthroplasty: who is afraid of outpatient surgery?[J].Knee Surg Sports Traumatol Arthrosc.201725(3):759-766

26. Kort NP, Bemelmans YFL, Schotanus MGM, Outpatient surgery for unicompartmental knee arthroplasty is effective and safe.[J].Knee Surg Sports Traumatol Arthrosc.201725(9):2659-2667

27. Turner JD, Henshaw DS, Weller RS, et al.Perineural dexamethasone successfully prolongs adductor canal block when assessed by objective pinprick sensory testing: A prospective randomized dosedependent placebo-controlled equivalency trial.[J].J Clin Anesth.201848():51-57

28. Baldawi M, McKelvey G, Saasouh W, et al.A Comparison of Neuraxial and General Anesthesia for Thirty-Day Postoperative Outcomes in United States Veterans Undergoing Total Knee Arthroplasty. [J].J Arthroplasty.2020():

29. Chaturvedi R, Burton BN, Gabriel RA, Complication Rates and the Benefits of Neuraxial Anesthesia in the Patient With High Comorbidity Burden Undergoing Primary Total Joint Arthroplasty.[J].J Arthroplasty.2020():

\section{Figures}



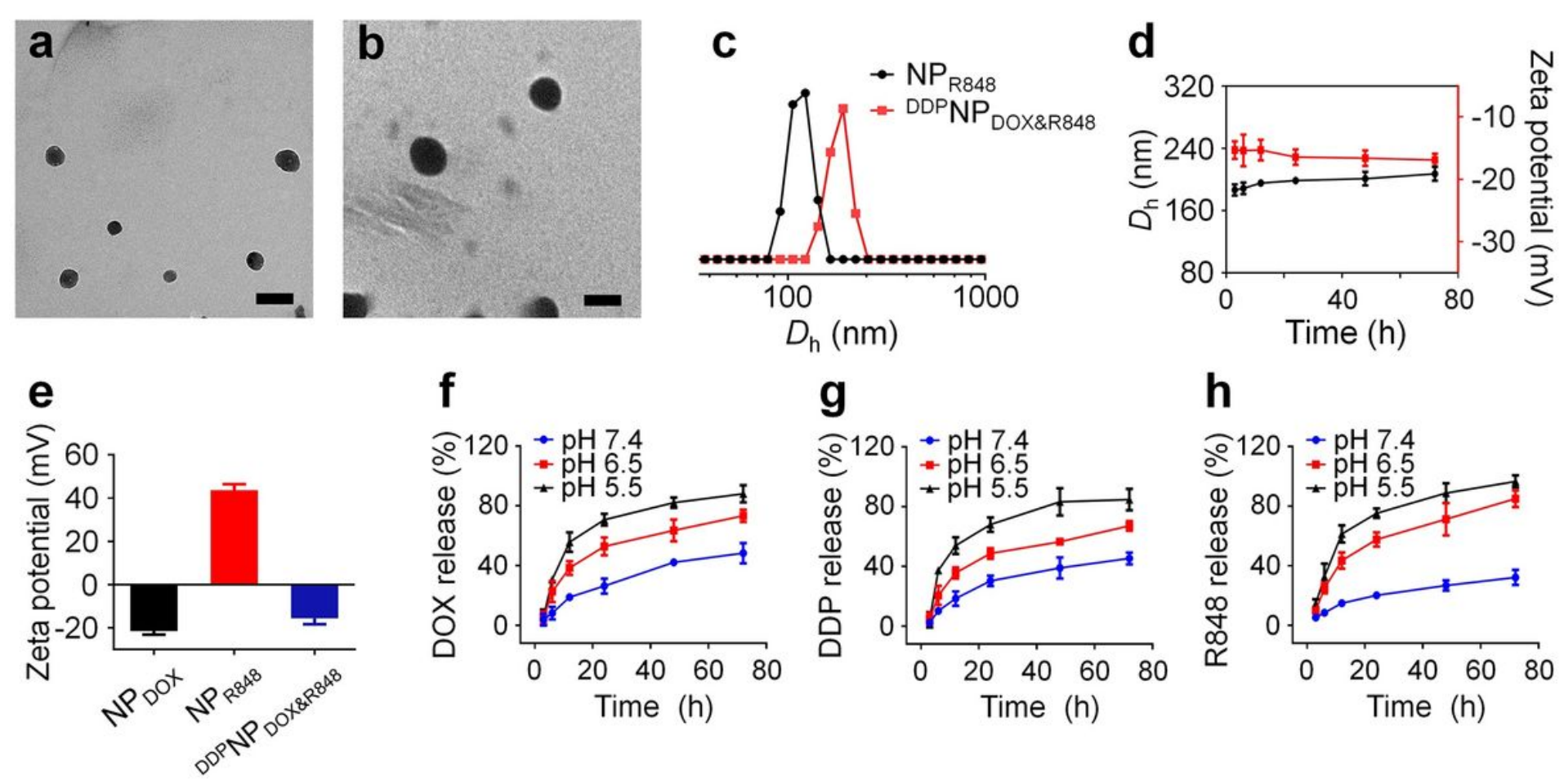

\section{Figure 1}

Characterization of DDPNPDOX\&R848 TEM images of a NPR848 and b DDPNPDOX\&R848. Scale bars: $200 \mathrm{~nm}$. c Size distribution of NPR848 and DDPNPDOX\&R848. d Size and zata potential changes of DDPNPDOX\&R848 in 10\% FBS solution over different times. e Zeta potential of NPDOX, NPR848, and DDPNPDOX\&R848. $\mathrm{f}$ - $\mathrm{h}$ Cumulative drug release from DDPNPDOX\&R848 under different $\mathrm{pH}$ values (7.4, 6.5, and 5.5).
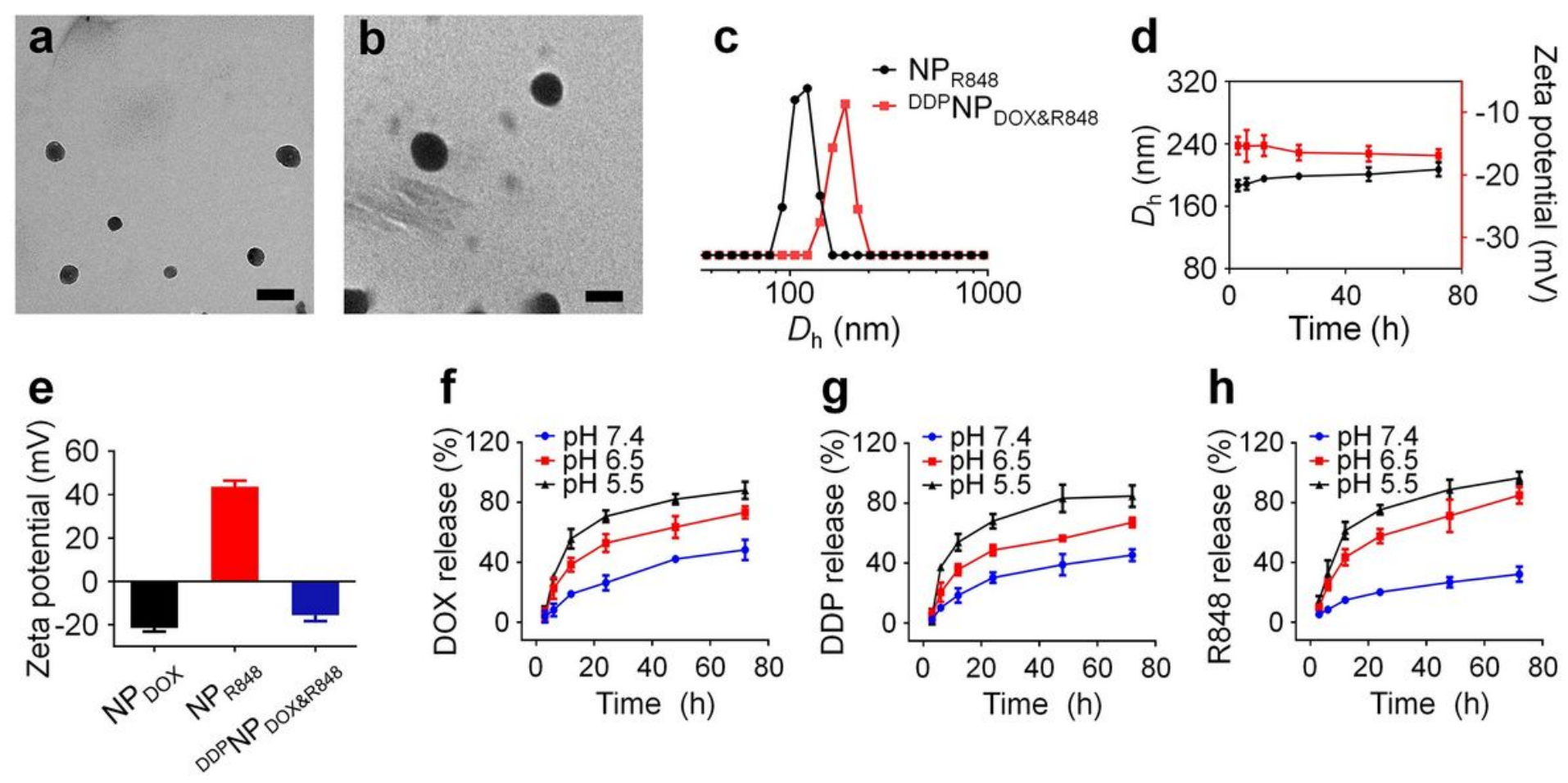
Figure 1

Characterization of DDPNPDOX\&R848 TEM images of a NPR848 and b DDPNPDOX\&R848. Scale bars: $200 \mathrm{~nm}$. c Size distribution of NPR848 and DDPNPDOX\&R848. d Size and zata potential changes of DDPNPDOX\&R848 in 10\% FBS solution over different times. e Zeta potential of NPDOX, NPR848, and DDPNPDOX\&R848. $\mathrm{f}$ - $\mathrm{h}$ Cumulative drug release from DDPNPDOX\&R848 under different $\mathrm{pH}$ values (7.4, 6.5, and 5.5).
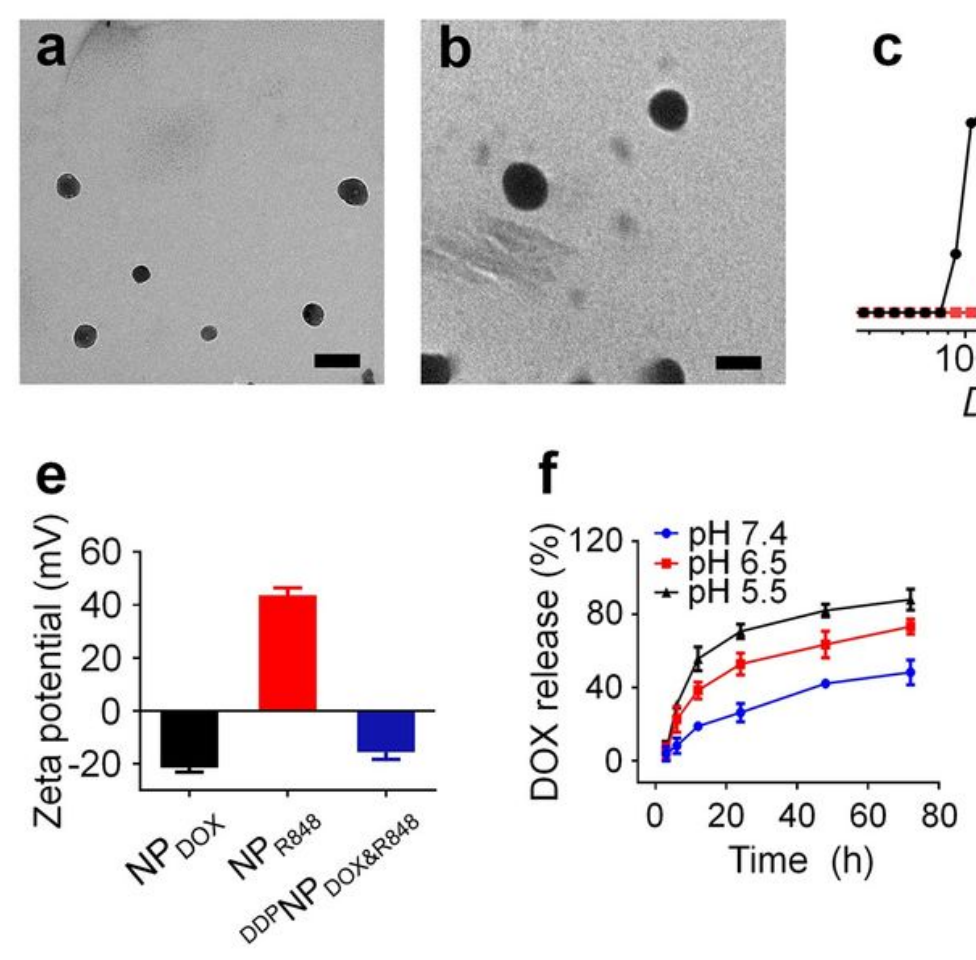

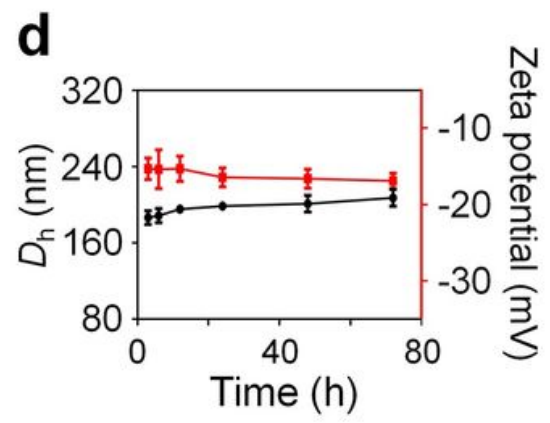

Figure 1

Characterization of DDPNPDOX\&R848 TEM images of a NPR848 and b DDPNPDOX\&R848. Scale bars: $200 \mathrm{~nm}$. c Size distribution of NPR848 and DDPNPDOX\&R848. d Size and zata potential changes of DDPNPDOX\&R848 in 10\% FBS solution over different times. e Zeta potential of NPDOX, NPR848, and DDPNPDOX\&R848. $\mathrm{f}$ - $\mathrm{h}$ Cumulative drug release from DDPNPDOX\&R848 under different $\mathrm{pH}$ values (7.4, 6.5, and 5.5). 

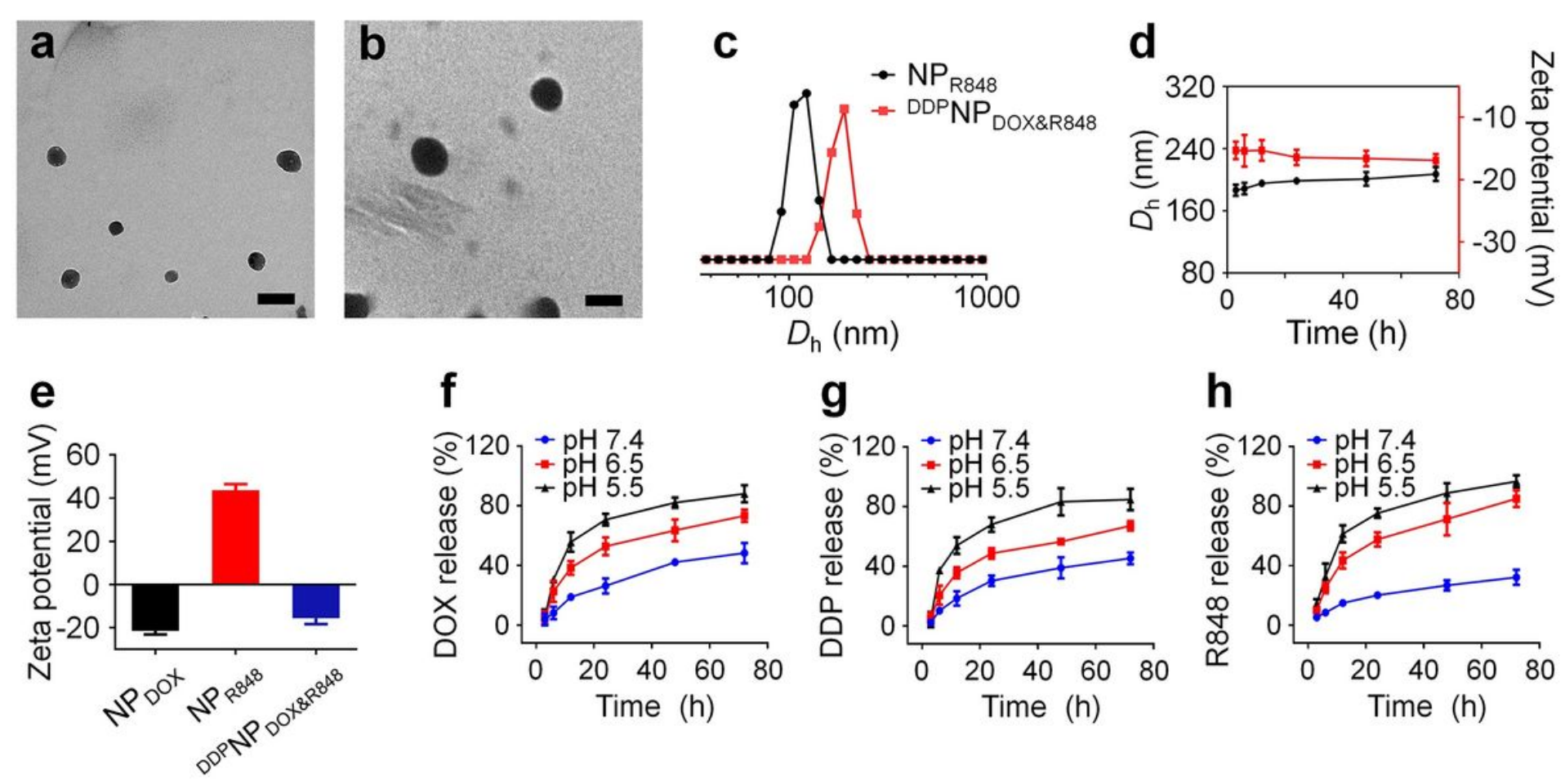

\section{Figure 1}

Characterization of DDPNPDOX\&R848 TEM images of a NPR848 and b DDPNPDOX\&R848. Scale bars: $200 \mathrm{~nm}$. c Size distribution of NPR848 and DDPNPDOX\&R848. d Size and zata potential changes of DDPNPDOX\&R848 in 10\% FBS solution over different times. e Zeta potential of NPDOX, NPR848, and DDPNPDOX\&R848. $\mathrm{f}$ - $\mathrm{h}$ Cumulative drug release from DDPNPDOX\&R848 under different $\mathrm{pH}$ values (7.4, 6.5, and 5.5).
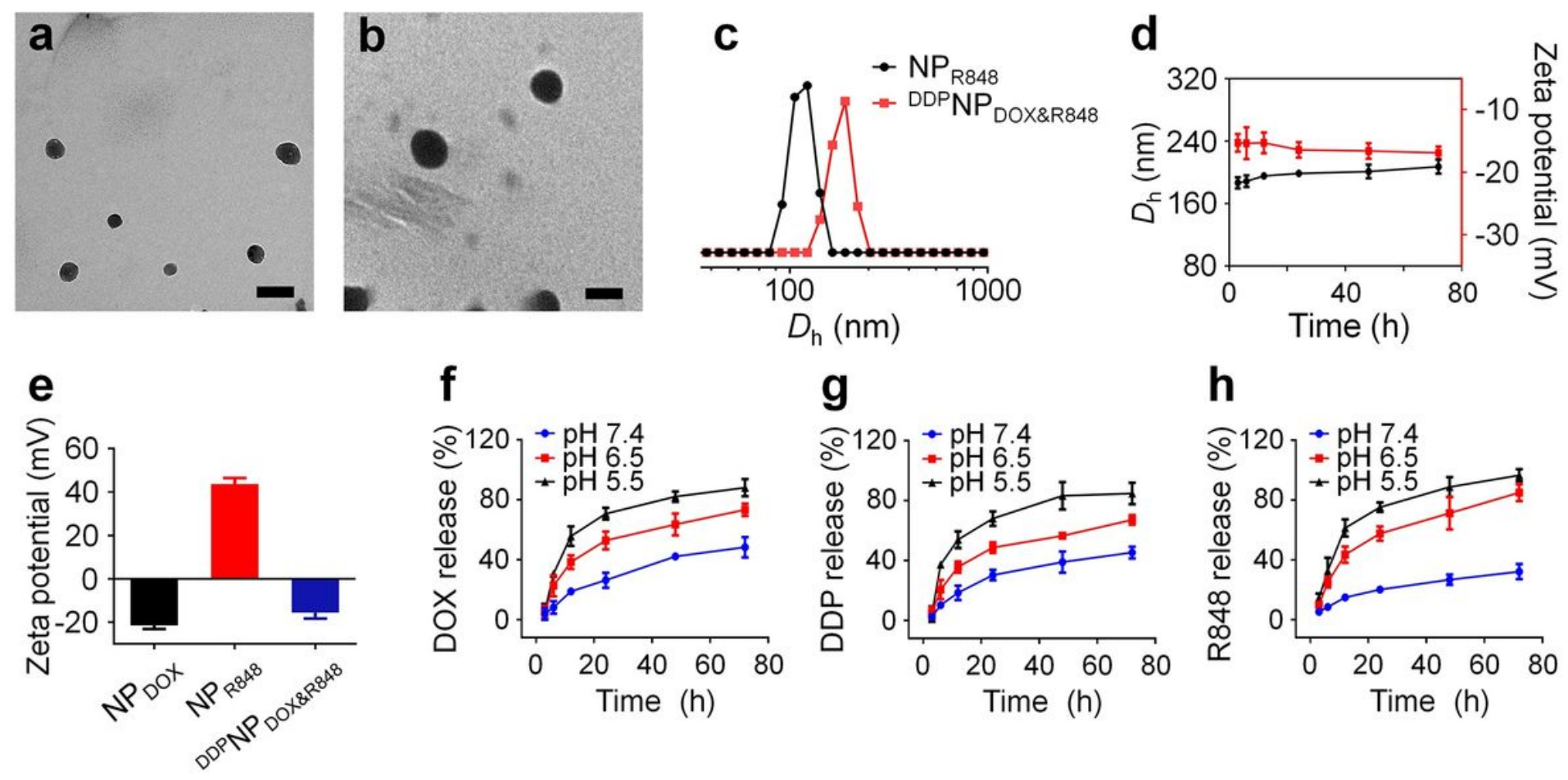
Figure 1

Characterization of DDPNPDOX\&R848 TEM images of a NPR848 and b DDPNPDOX\&R848. Scale bars: $200 \mathrm{~nm}$. c Size distribution of NPR848 and DDPNPDOX\&R848. d Size and zata potential changes of DDPNPDOX\&R848 in 10\% FBS solution over different times. e Zeta potential of NPDOX, NPR848, and DDPNPDOX\&R848. $\mathrm{f}$ - $\mathrm{h}$ Cumulative drug release from DDPNPDOX\&R848 under different $\mathrm{pH}$ values (7.4, 6.5 , and 5.5).

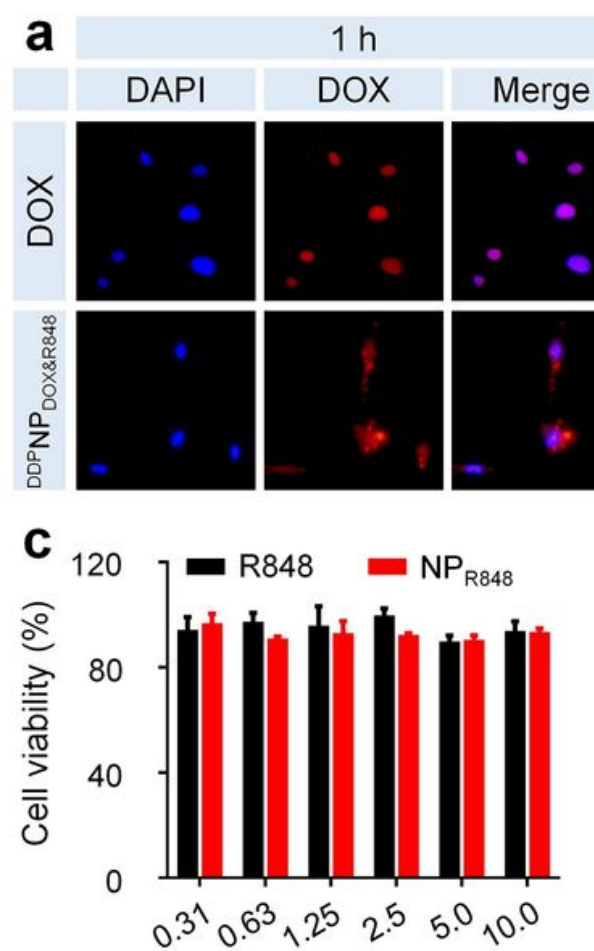

Concentration of R848 $\left(\mu \mathrm{g} \mathrm{mL}^{-1}\right)$

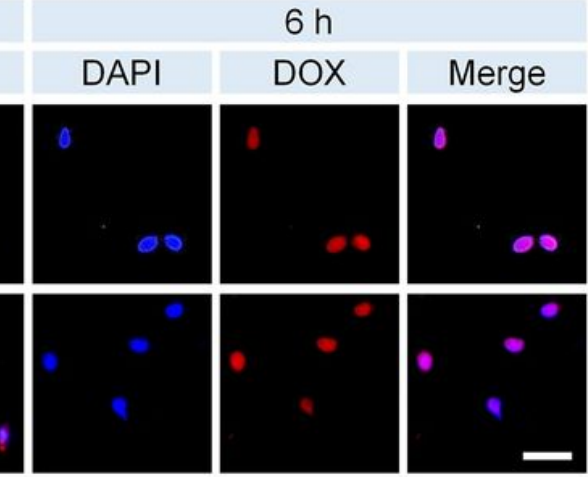

d

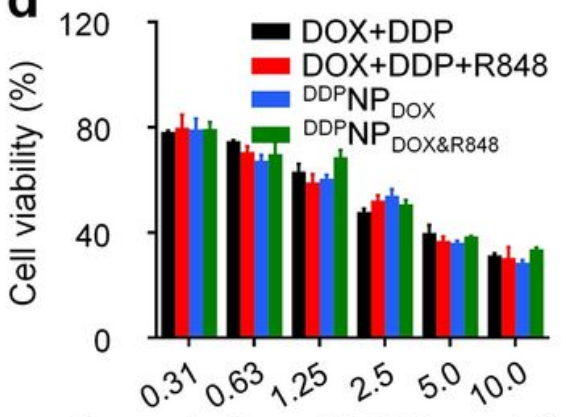

Concentration of DOX $\left(\mu \mathrm{g} \mathrm{mL}^{-1}\right)$
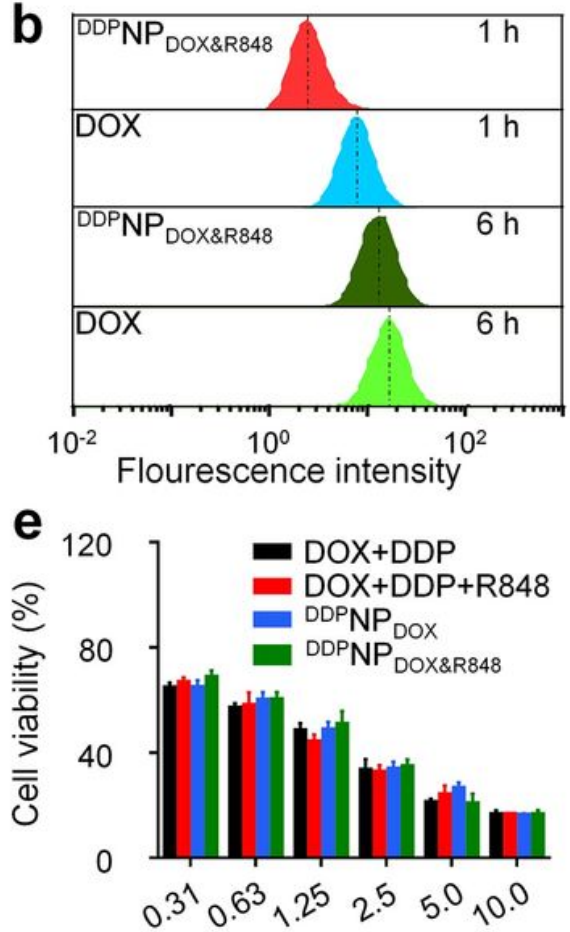

Concentration of DOX $(\mu \mathrm{g} \mathrm{mL}-1)$

\section{Figure 2}

Tumor cell uptake and cytotoxicity a Fluorescence images and b flow cytometry analysis of K7M2 osteosarcoma cells after incubation with DOX or DDPNPDOX\&R848 for 1 and $6 \mathrm{~h}$. Scale bars: $200 \mu \mathrm{m}$. C Cell viability of K7M2 osteosarcoma cells after treatment with R848 or pHis/R848 at various R848 concentrations after $48 \mathrm{~h}$ of incubation. Cell viability of K7M2 osteosarcoma cells after treatment with DOX + DDP, DOX + DDP + R848, DDPNPDOX, or DDPNPDOX\&R848 at various DOX concentrations after d $24 \mathrm{~h}$ and e $48 \mathrm{~h}$, respectively. 


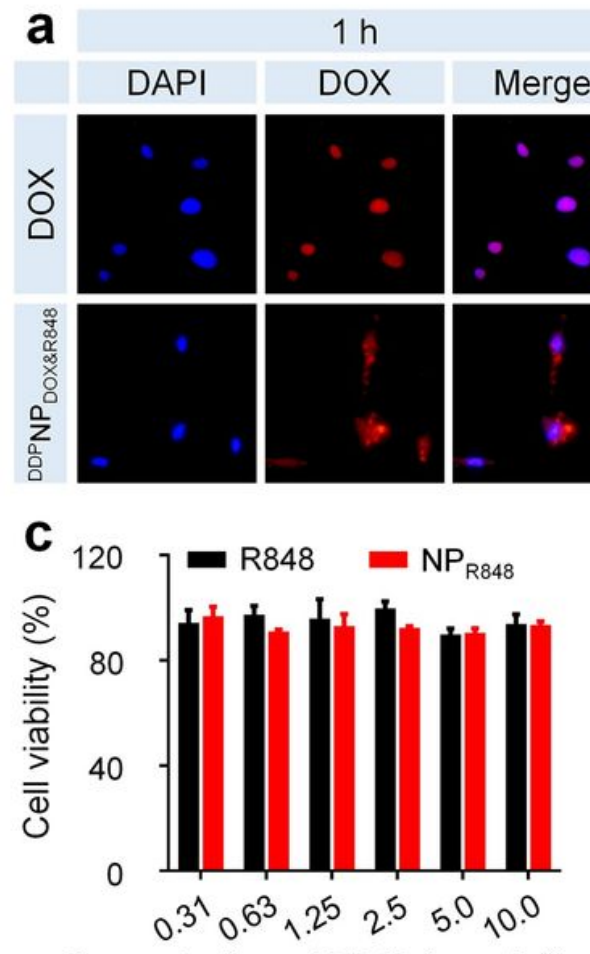

Concentration of R848 $\left(\mu \mathrm{g} \mathrm{mL}^{-1}\right)$
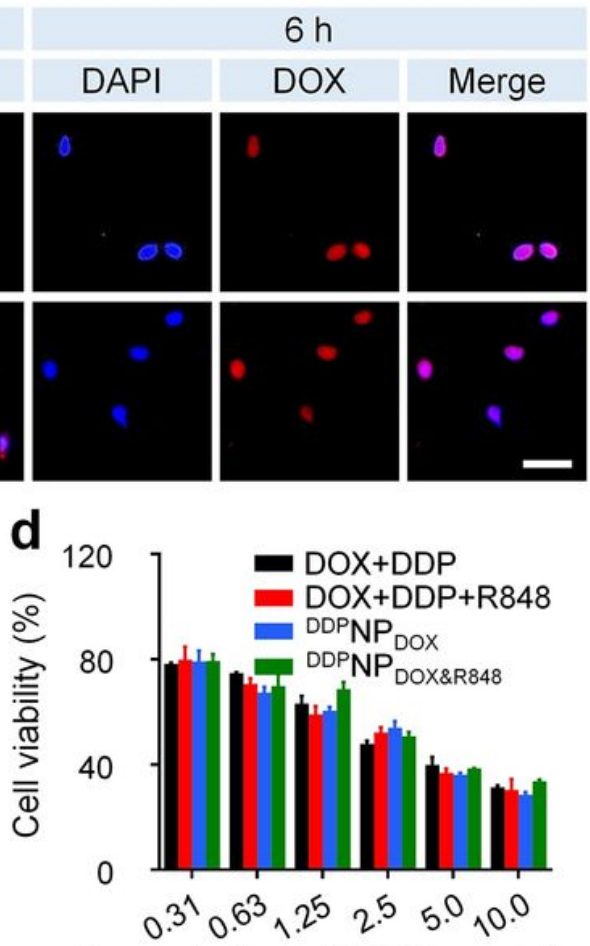

Concentration of DOX $(\mu \mathrm{g} \mathrm{mL}-1)$
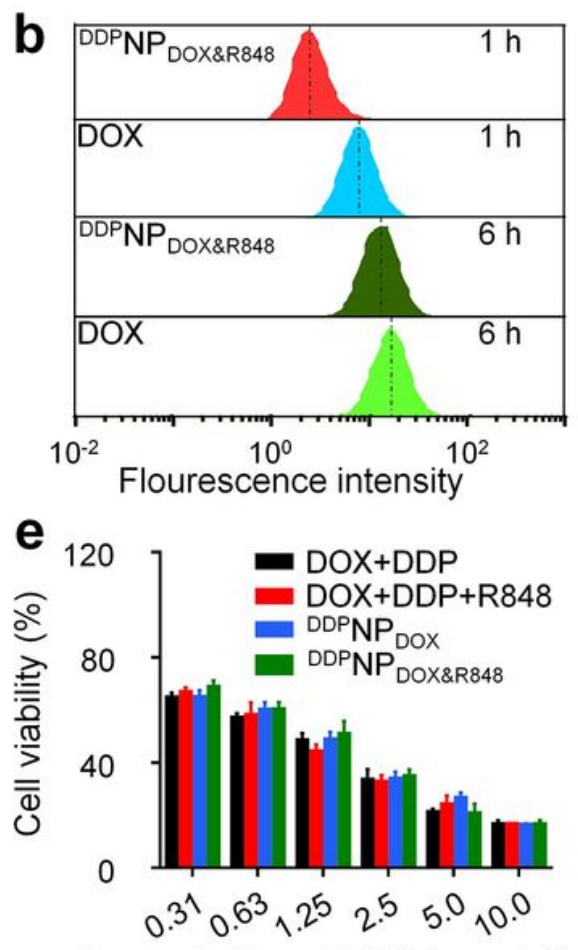

Concentration of DOX $\left(\mu \mathrm{g} \mathrm{mL} \mathrm{L}^{-1}\right)$

\section{Figure 2}

Tumor cell uptake and cytotoxicity a Fluorescence images and b flow cytometry analysis of K7M2 osteosarcoma cells after incubation with DOX or DDPNPDOX\&R848 for 1 and 6 h. Scale bars: $200 \mu \mathrm{m}$. C Cell viability of K7M2 osteosarcoma cells after treatment with R848 or pHis/R848 at various R848 concentrations after $48 \mathrm{~h}$ of incubation. Cell viability of K7M2 osteosarcoma cells after treatment with DOX + DDP, DOX + DDP + R848, DDPNPDOX, or DDPNPDOX\&R848 at various DOX concentrations after d $24 \mathrm{~h}$ and e $48 \mathrm{~h}$, respectively. 


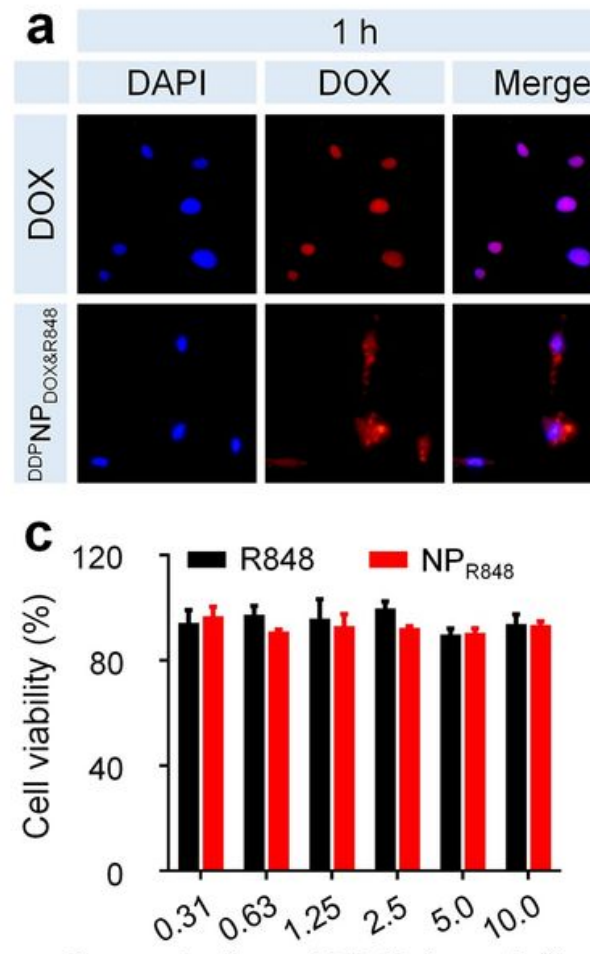

Concentration of R848 $\left(\mu \mathrm{g} \mathrm{mL}^{-1}\right)$
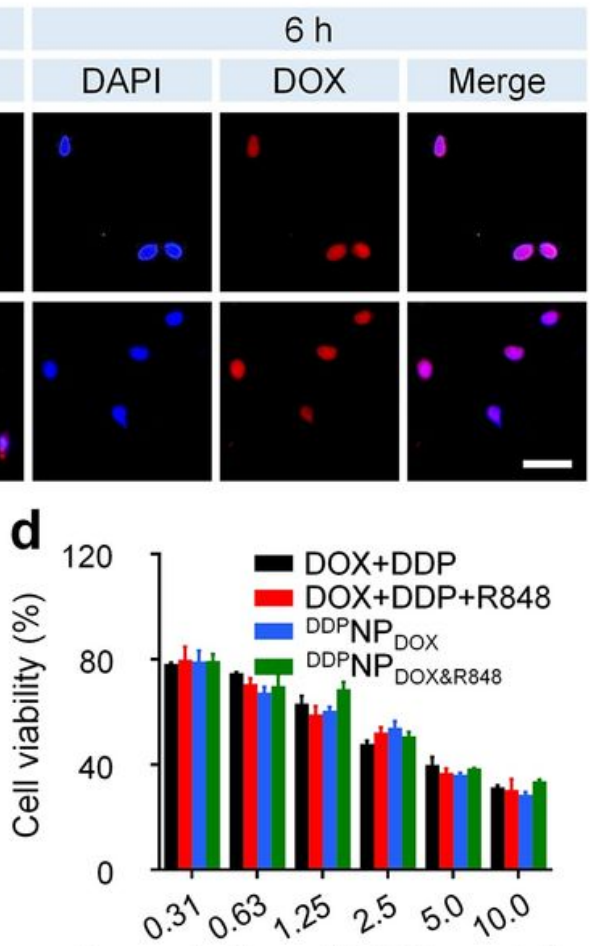

Concentration of DOX $(\mu \mathrm{g} \mathrm{mL}-1)$
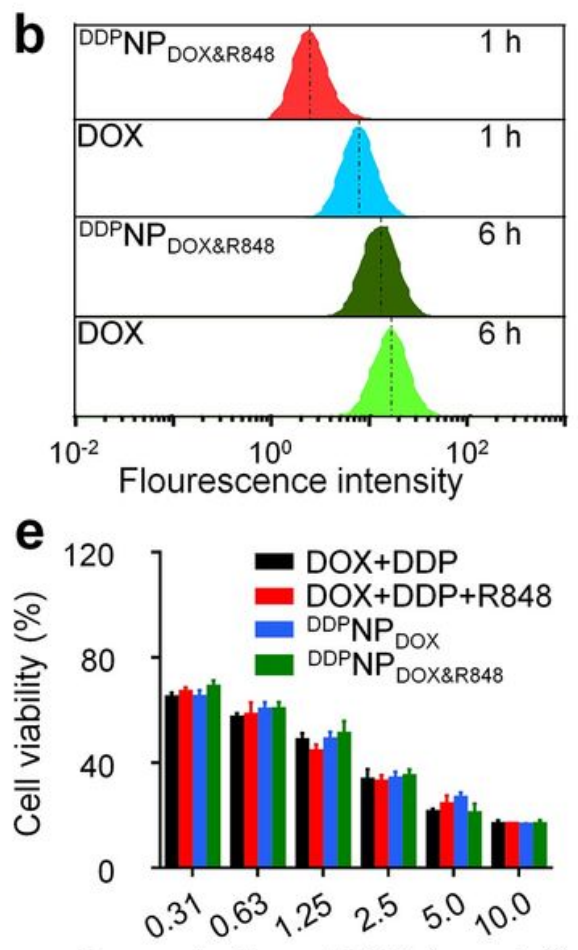

Concentration of DOX $\left(\mu \mathrm{g} \mathrm{mL} \mathrm{L}^{-1}\right)$

\section{Figure 2}

Tumor cell uptake and cytotoxicity a Fluorescence images and b flow cytometry analysis of K7M2 osteosarcoma cells after incubation with DOX or DDPNPDOX\&R848 for 1 and 6 h. Scale bars: $200 \mu \mathrm{m}$. C Cell viability of K7M2 osteosarcoma cells after treatment with R848 or pHis/R848 at various R848 concentrations after $48 \mathrm{~h}$ of incubation. Cell viability of K7M2 osteosarcoma cells after treatment with DOX + DDP, DOX + DDP + R848, DDPNPDOX, or DDPNPDOX\&R848 at various DOX concentrations after d $24 \mathrm{~h}$ and e $48 \mathrm{~h}$, respectively. 


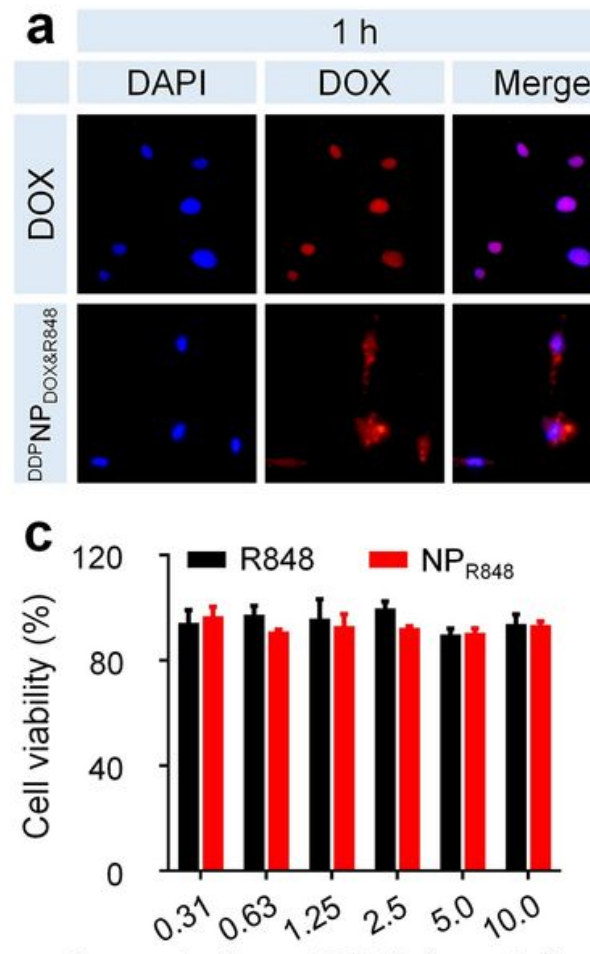

Concentration of R848 $\left(\mu \mathrm{g} \mathrm{mL}^{-1}\right)$
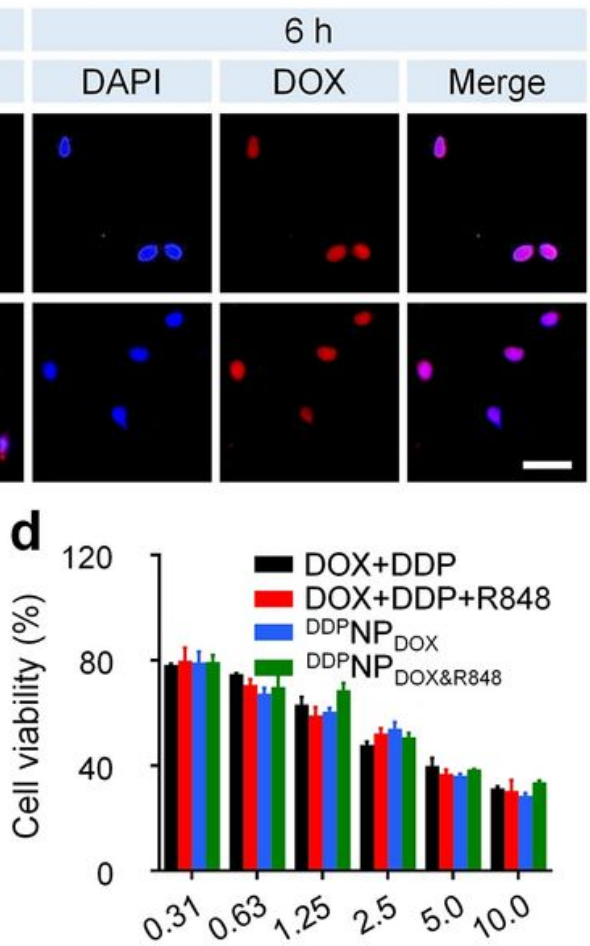

Concentration of DOX $(\mu \mathrm{g} \mathrm{mL}-1)$
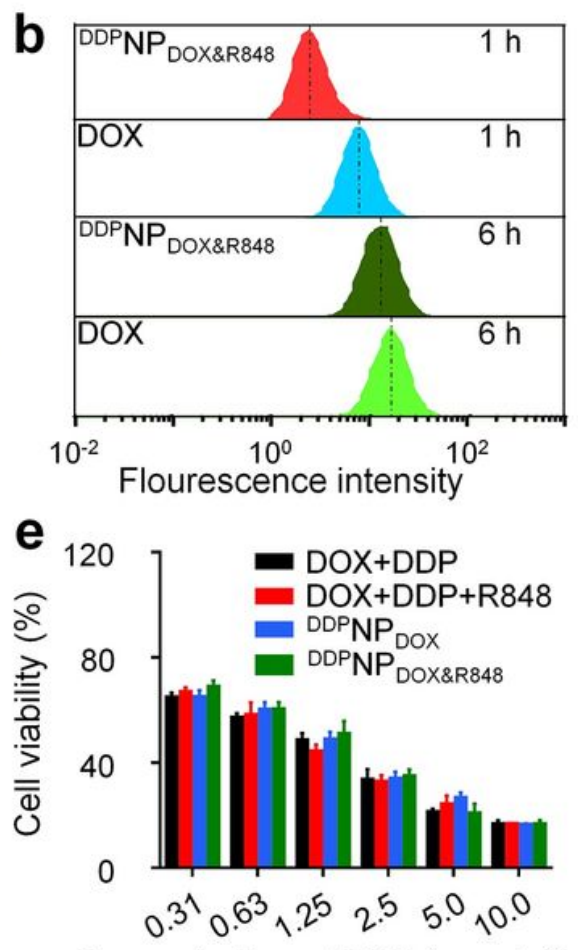

Concentration of DOX $\left(\mu \mathrm{g} \mathrm{mL} \mathrm{m}^{-1}\right)$

\section{Figure 2}

Tumor cell uptake and cytotoxicity a Fluorescence images and b flow cytometry analysis of K7M2 osteosarcoma cells after incubation with DOX or DDPNPDOX\&R848 for 1 and 6 h. Scale bars: $200 \mu \mathrm{m}$. C Cell viability of K7M2 osteosarcoma cells after treatment with R848 or pHis/R848 at various R848 concentrations after $48 \mathrm{~h}$ of incubation. Cell viability of K7M2 osteosarcoma cells after treatment with DOX + DDP, DOX + DDP + R848, DDPNPDOX, or DDPNPDOX\&R848 at various DOX concentrations after d $24 \mathrm{~h}$ and e $48 \mathrm{~h}$, respectively. 
a

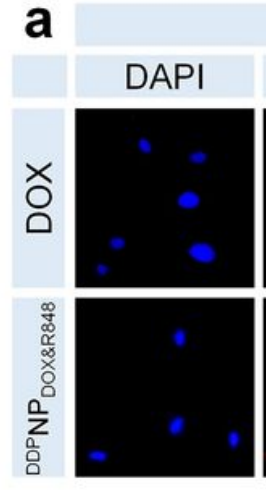

$1 \mathrm{~h}$
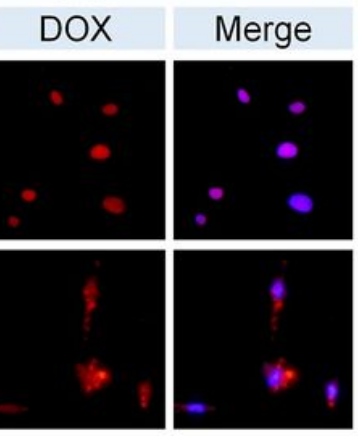

C

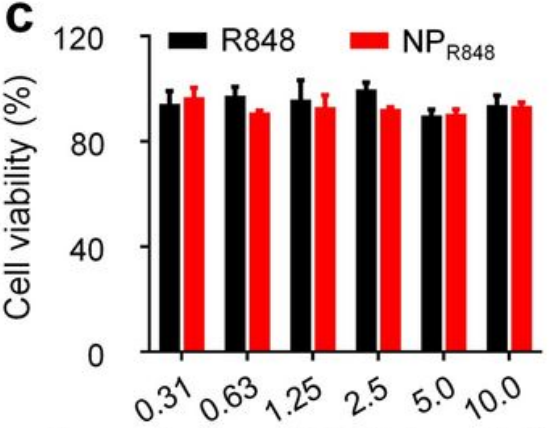

Concentration of R848 ( $\left.\mu \mathrm{g} \mathrm{mL}^{-1}\right)$
$6 \mathrm{~h}$

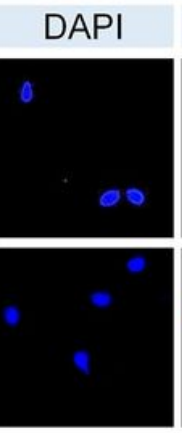

DOX
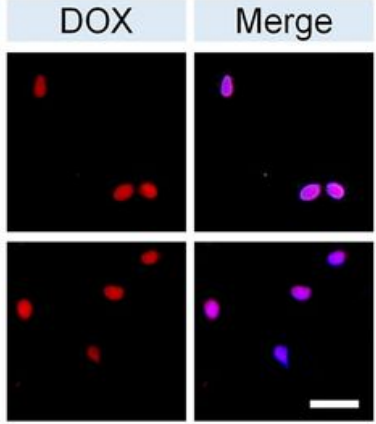

d
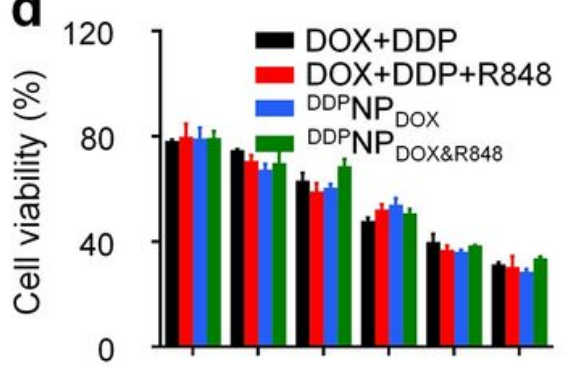

$0.3^{1} 0.6^{3} \wedge .2^{5} \quad 2.5 \quad 5.0,10.0$

Concentration of DOX $\left(\mu \mathrm{g} \mathrm{mL}^{-1}\right)$ b
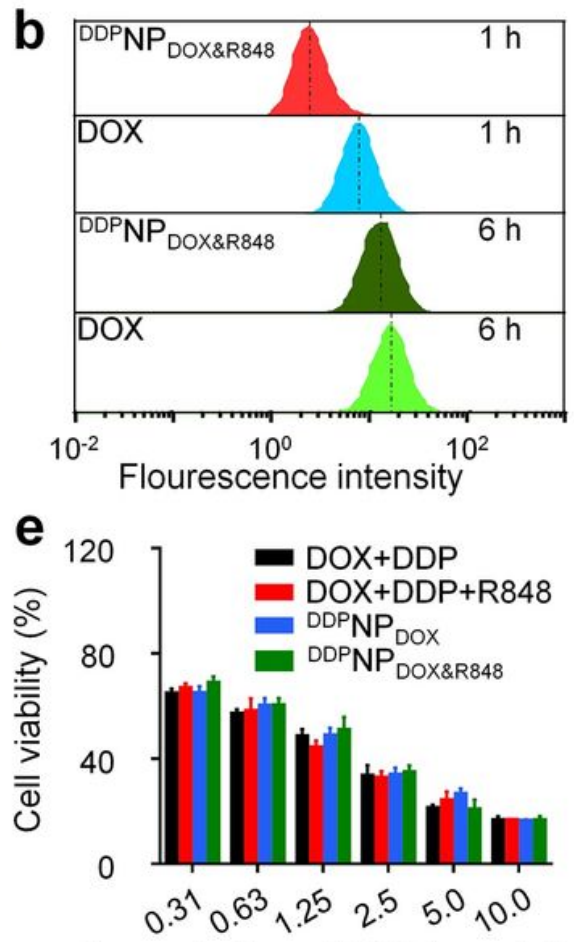

Concentration of DOX $\left(\mu \mathrm{g} \mathrm{mL} \mathrm{L}^{-1}\right)$

\section{Figure 2}

Tumor cell uptake and cytotoxicity a Fluorescence images and b flow cytometry analysis of K7M2 osteosarcoma cells after incubation with DOX or DDPNPDOX\&R848 for 1 and 6 h. Scale bars: $200 \mu \mathrm{m}$. C Cell viability of K7M2 osteosarcoma cells after treatment with R848 or pHis/R848 at various R848 concentrations after $48 \mathrm{~h}$ of incubation. Cell viability of K7M2 osteosarcoma cells after treatment with DOX + DDP, DOX + DDP + R848, DDPNPDOX, or DDPNPDOX\&R848 at various DOX concentrations after d $24 \mathrm{~h}$ and e $48 \mathrm{~h}$, respectively. 

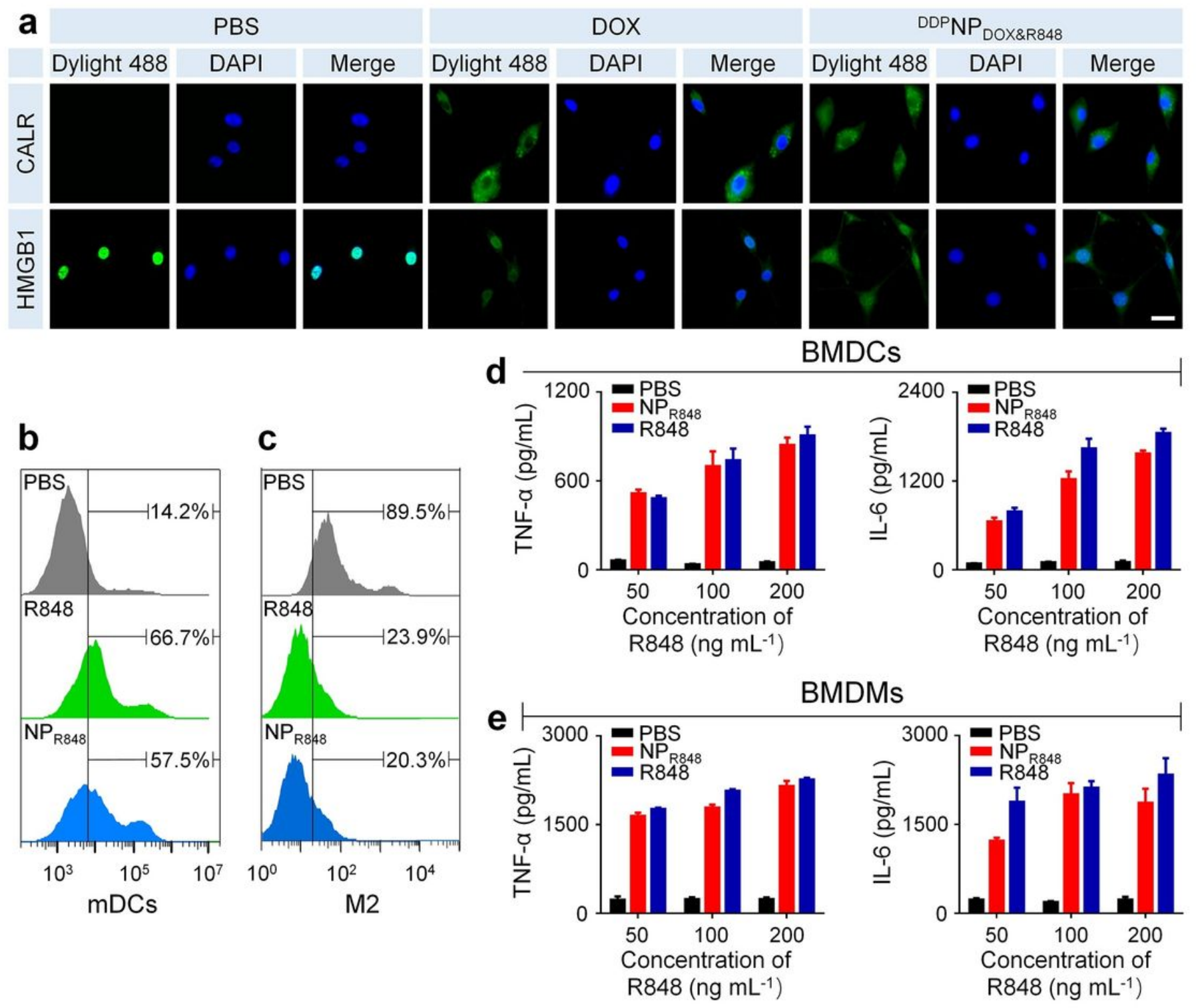

Figure 3

ICD induction and immune cell reeducation a Immunofluorescence of CALR and HMGB1 in K7M2 osteosarcoma cells after incubation with DOX or DDPNPDOX\&R848 for $12 \mathrm{~h}$. Scale bars: $60 \mu \mathrm{m}$. Flow cytometry quantification of b mDCs (CD11c+ CD86+) and c M2 macrophages (F4/80+ CD206+) after incubation of BMDCs or BMDMs with R848 or pHis/R848 for $24 \mathrm{~h}$. The production of proinflammatory cytokines (TNF- $\alpha$ and IL-6) in cell supernatants of $d$ BMDCs and e BMDMs was analyzed by ELISA. 

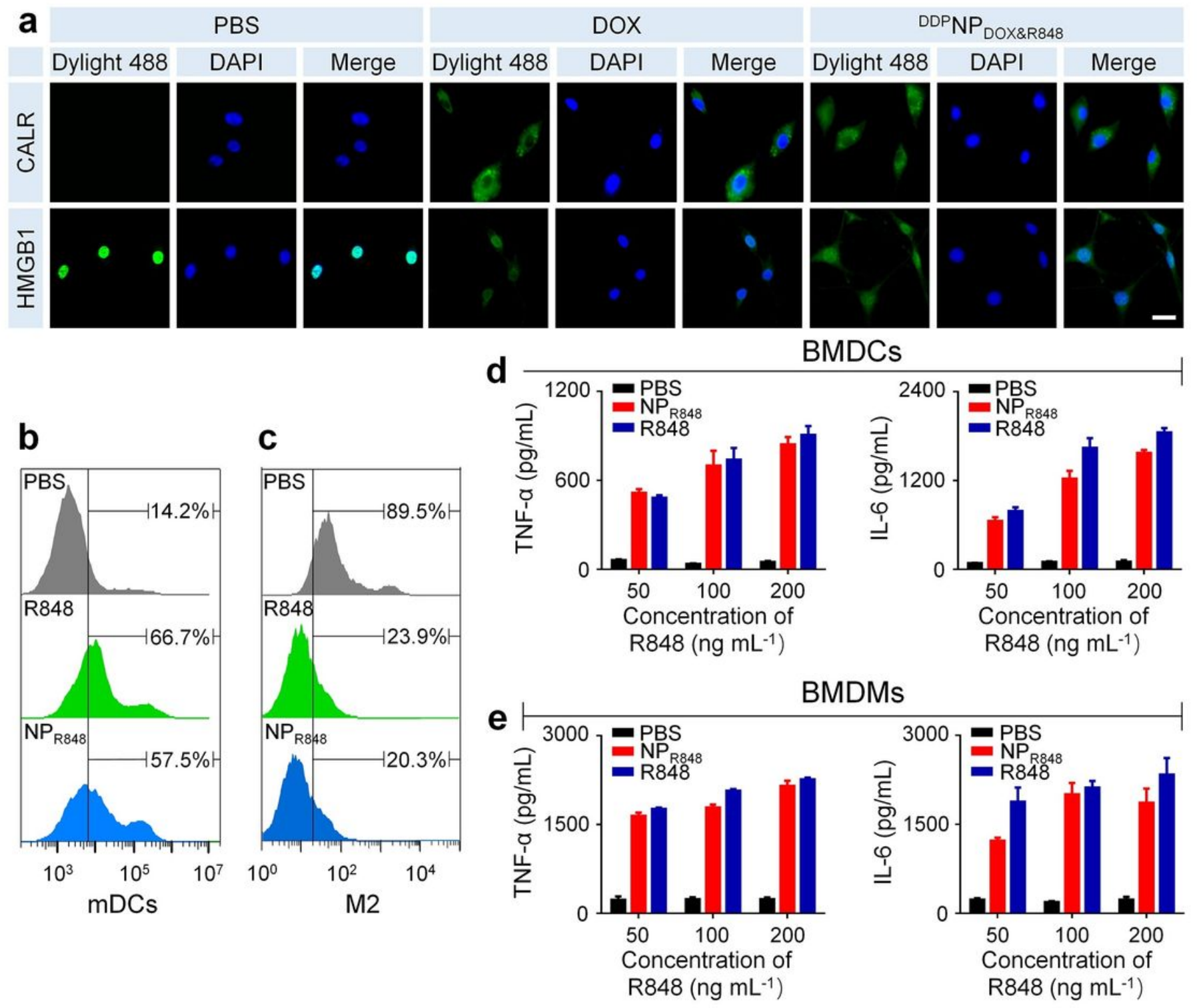

Figure 3

ICD induction and immune cell reeducation a Immunofluorescence of CALR and HMGB1 in K7M2 osteosarcoma cells after incubation with DOX or DDPNPDOX\&R848 for $12 \mathrm{~h}$. Scale bars: $60 \mu \mathrm{m}$. Flow cytometry quantification of b mDCs (CD11c+ CD86+) and c M2 macrophages (F4/80+ CD206+) after incubation of BMDCs or BMDMs with R848 or pHis/R848 for $24 \mathrm{~h}$. The production of proinflammatory cytokines (TNF- $\alpha$ and IL-6) in cell supernatants of $d$ BMDCs and e BMDMs was analyzed by ELISA. 

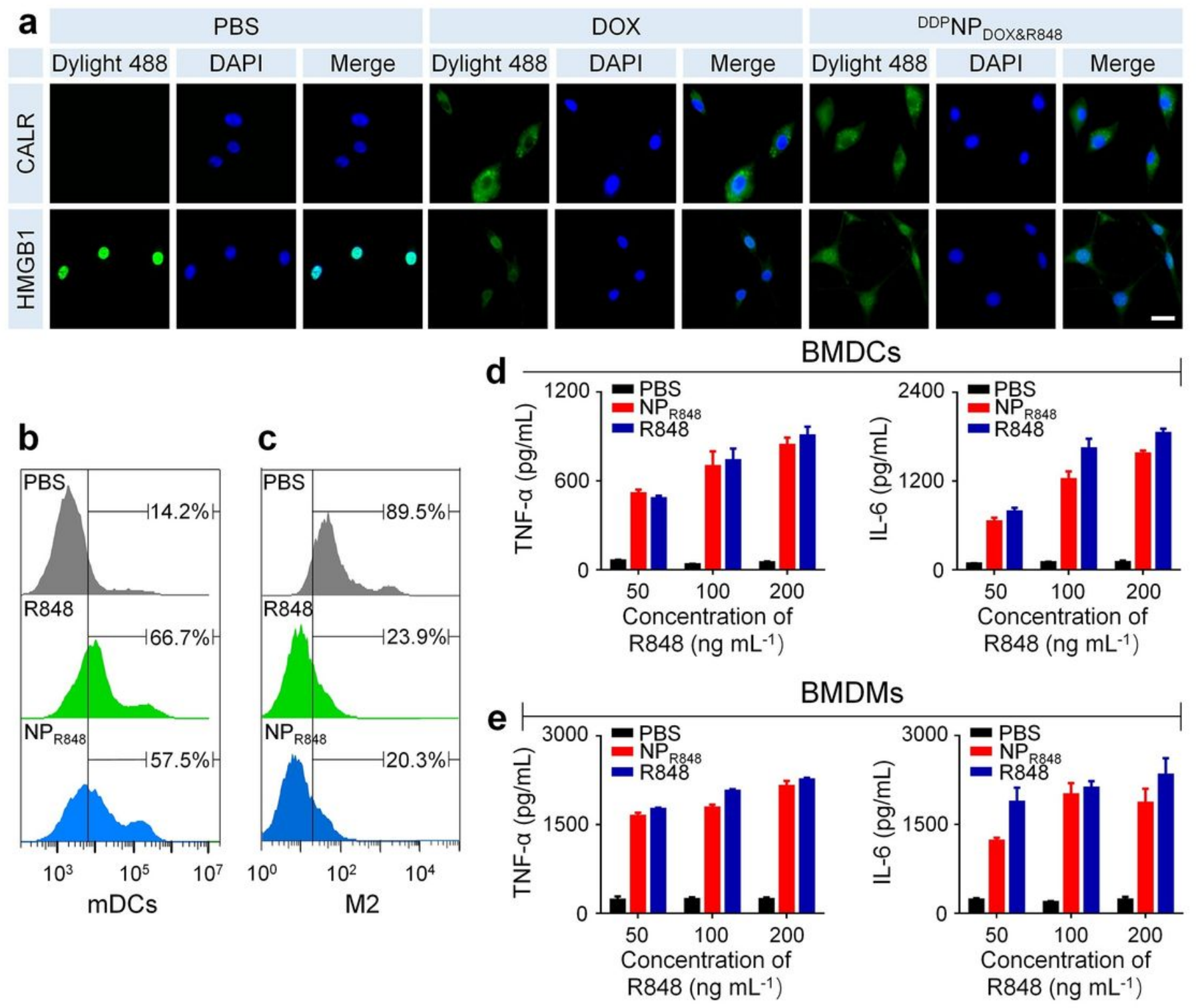

Figure 3

ICD induction and immune cell reeducation a Immunofluorescence of CALR and HMGB1 in K7M2 osteosarcoma cells after incubation with DOX or DDPNPDOX\&R848 for $12 \mathrm{~h}$. Scale bars: $60 \mu \mathrm{m}$. Flow cytometry quantification of b mDCs (CD11c+ CD86+) and c M2 macrophages (F4/80+ CD206+) after incubation of BMDCs or BMDMs with R848 or pHis/R848 for $24 \mathrm{~h}$. The production of proinflammatory cytokines (TNF- $\alpha$ and IL-6) in cell supernatants of $d$ BMDCs and e BMDMs was analyzed by ELISA. 

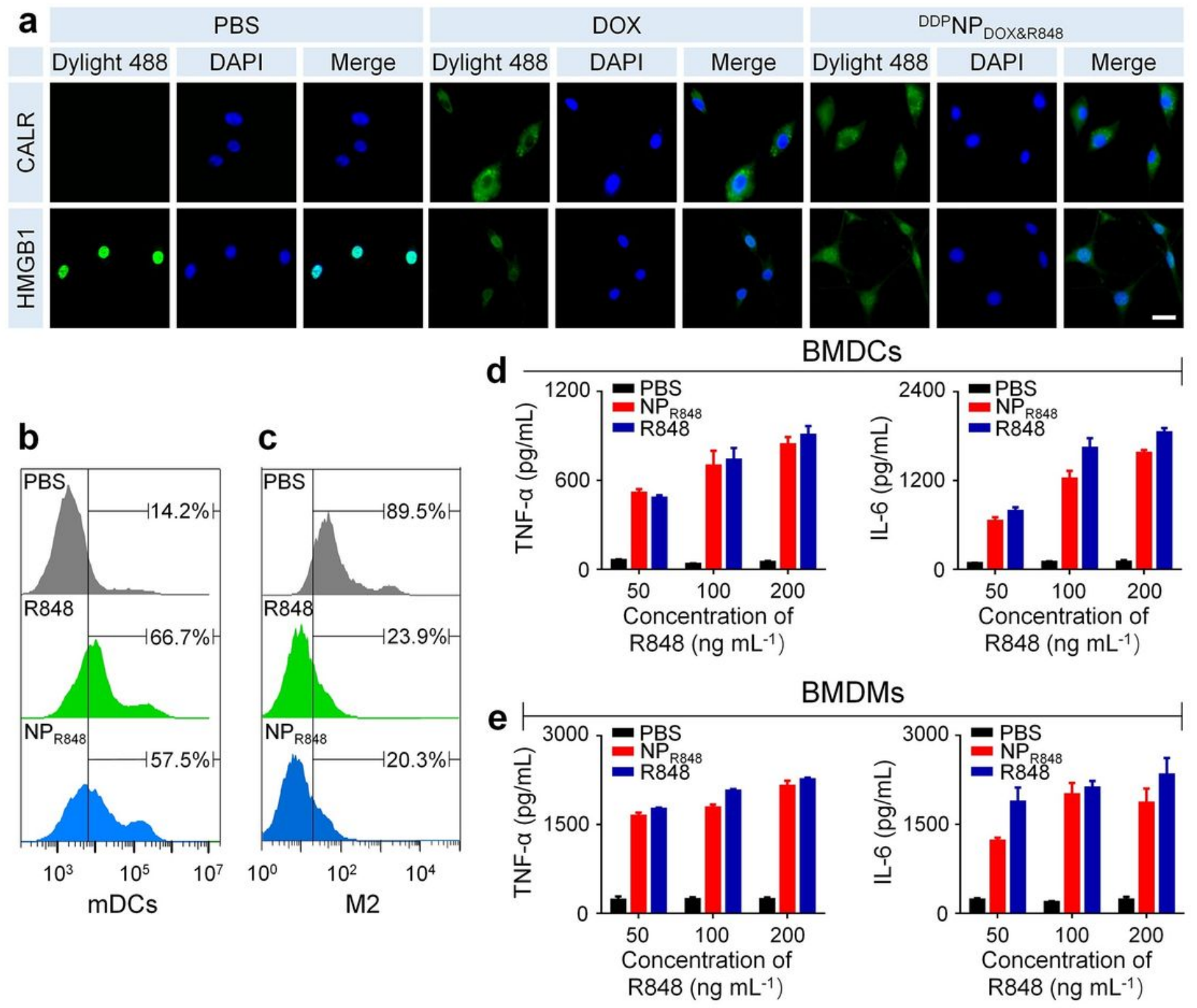

Figure 3

ICD induction and immune cell reeducation a Immunofluorescence of CALR and HMGB1 in K7M2 osteosarcoma cells after incubation with DOX or DDPNPDOX\&R848 for $12 \mathrm{~h}$. Scale bars: $60 \mu \mathrm{m}$. Flow cytometry quantification of b mDCs (CD11c+ CD86+) and c M2 macrophages (F4/80+ CD206+) after incubation of BMDCs or BMDMs with R848 or pHis/R848 for $24 \mathrm{~h}$. The production of proinflammatory cytokines (TNF- $\alpha$ and IL-6) in cell supernatants of $d$ BMDCs and e BMDMs was analyzed by ELISA. 

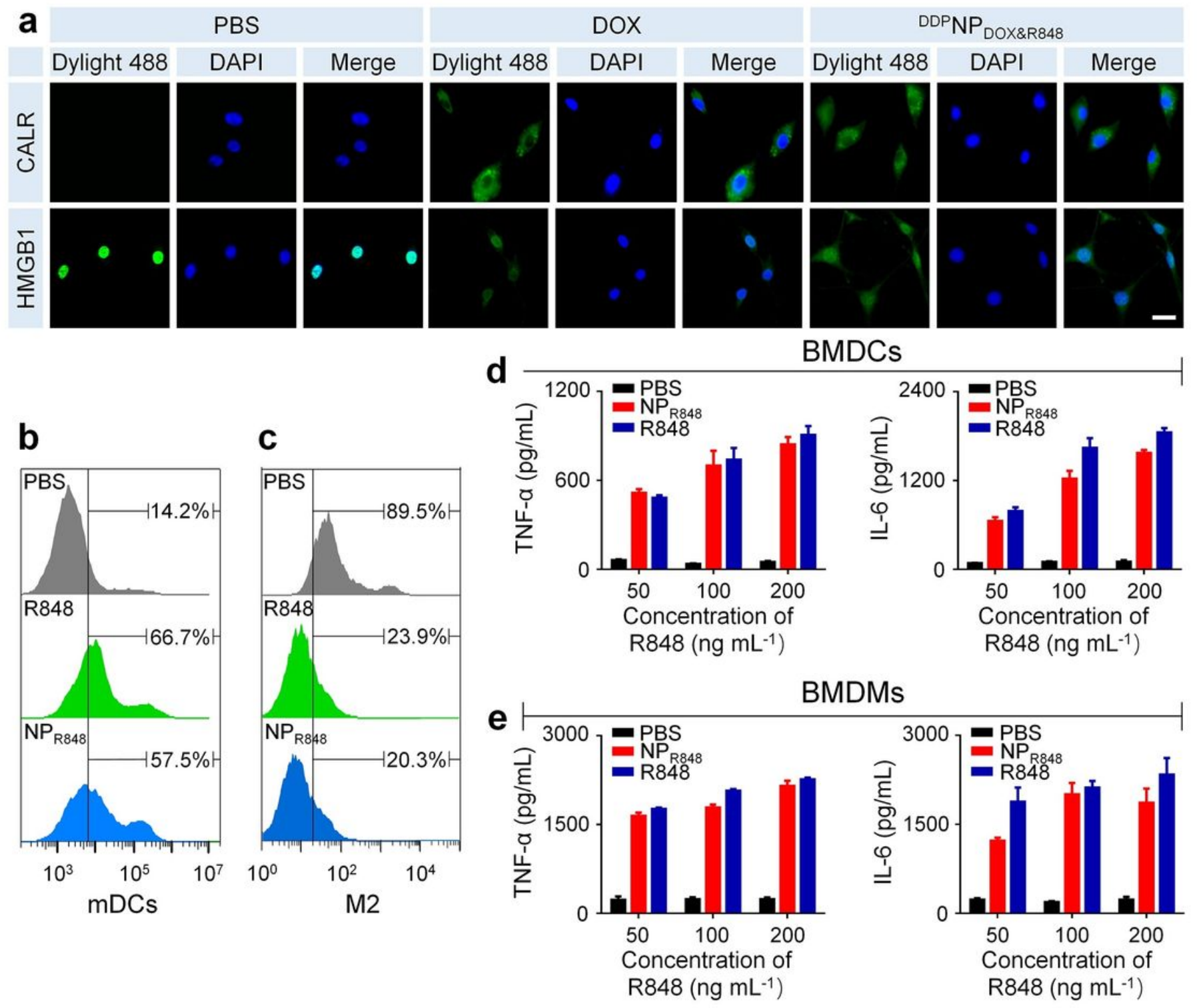

Figure 3

ICD induction and immune cell reeducation a Immunofluorescence of CALR and HMGB1 in K7M2 osteosarcoma cells after incubation with DOX or DDPNPDOX\&R848 for $12 \mathrm{~h}$. Scale bars: $60 \mu \mathrm{m}$. Flow cytometry quantification of b mDCs (CD11c+ CD86+) and c M2 macrophages (F4/80+ CD206+) after incubation of BMDCs or BMDMs with R848 or pHis/R848 for $24 \mathrm{~h}$. The production of proinflammatory cytokines (TNF- $\alpha$ and IL-6) in cell supernatants of $d$ BMDCs and e BMDMs was analyzed by ELISA. 
a
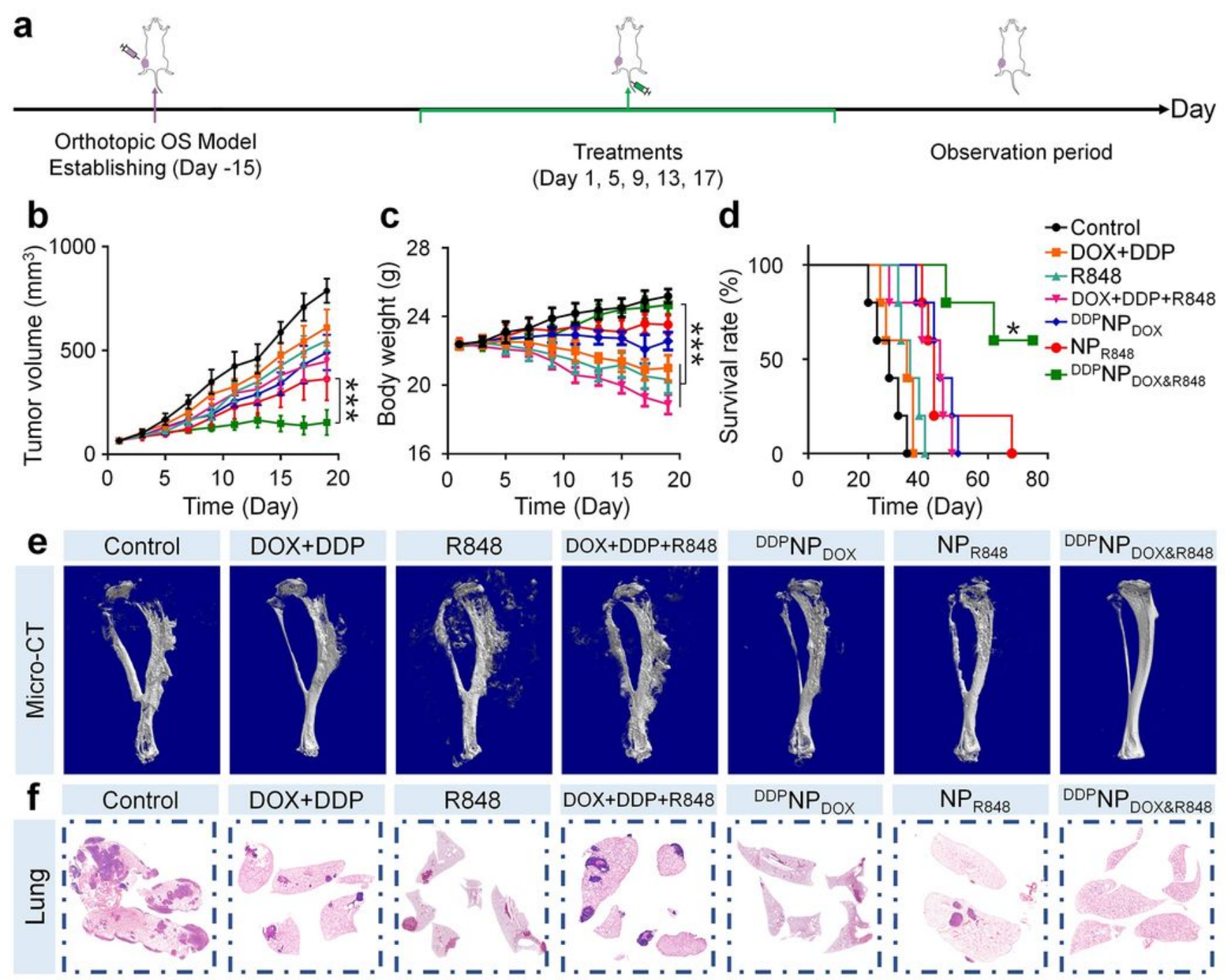

DDPNP DOX\&R848
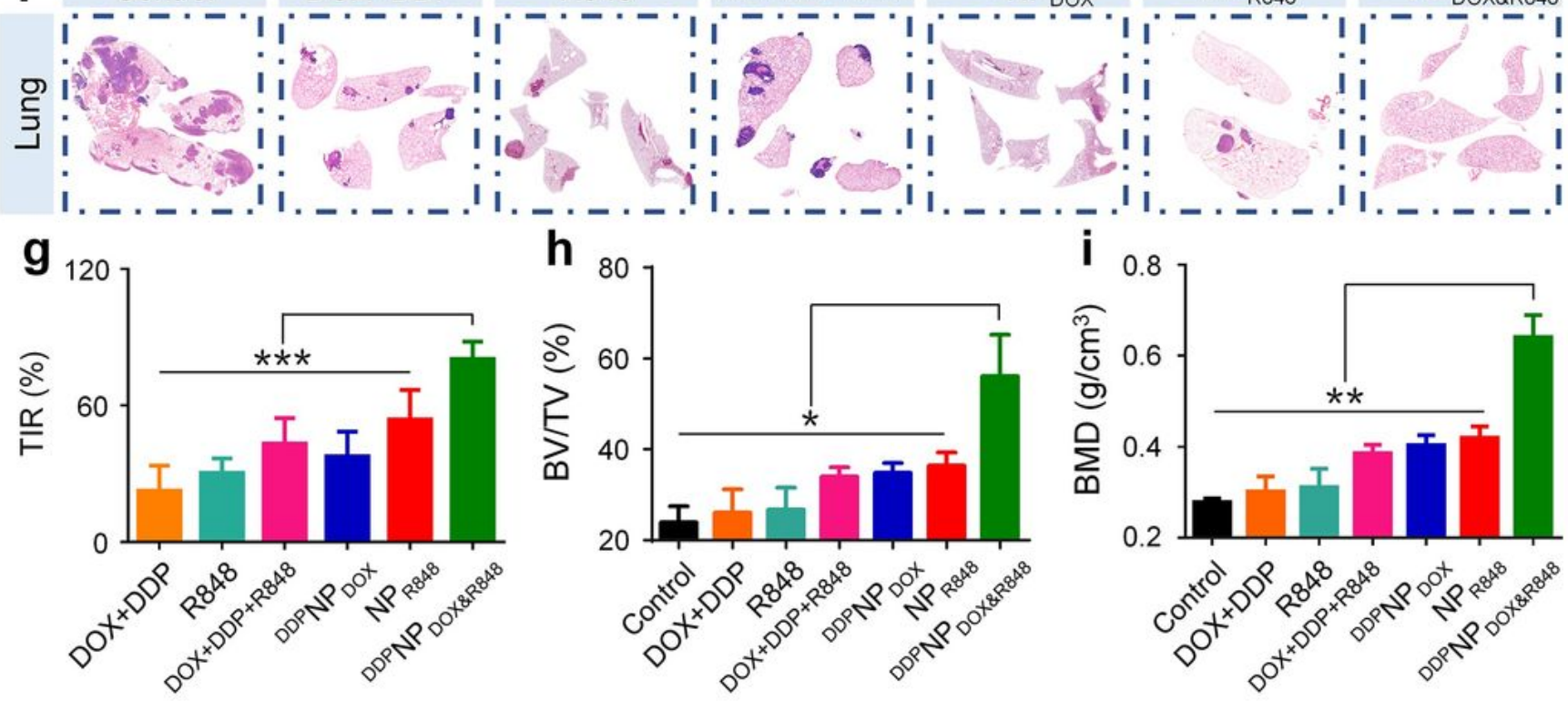

\section{Figure 4}

Antitumor effect in vivo a Experimental schedule for tumor induction and drug treatments. b Tumor volume, $c$ body weight, and d survival rate of mice after various treatments in seven groups. e 3D reconstructed images of the tibia in the orthotopic osteosarcoma inhibition experiment performed using micro-CT and $\mathrm{h} \mathrm{BV/TV}$ and $\mathrm{i}$ BMD in the ROls of the tibia in the orthotopic osteosarcoma inhibition experiment. $\mathrm{f} \mathrm{H \& E}$ staining analysis of lung metastasis in mice in different groups. g TIR of DOX + DDP, 
DOX + DDP + R848, DDPNPDOX, NPR848, and DDPNPDOX\&R848 in this experiment. Data are presented as the mean $\pm S D(n=8$ for $b-d$ and $g ; n=3$ for $h-i ; * P<0.05, * * P<0.01, * * * P<0.001)$.
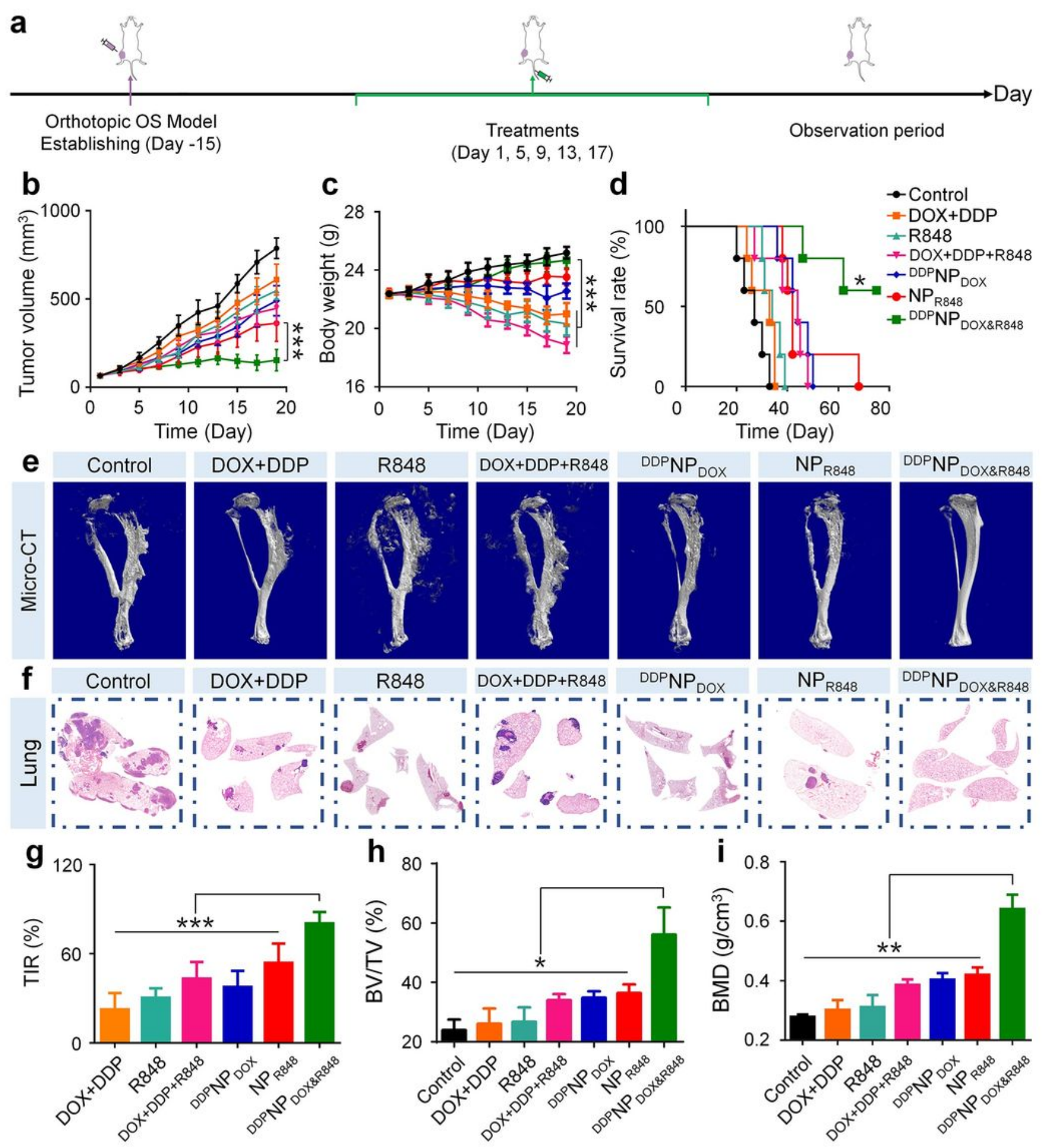

Figure 4

Antitumor effect in vivo a Experimental schedule for tumor induction and drug treatments. b Tumor volume, $c$ body weight, and d survival rate of mice after various treatments in seven groups. e 3D reconstructed images of the tibia in the orthotopic osteosarcoma inhibition experiment performed using 
micro-CT and $\mathrm{h} \mathrm{BV/TV}$ and $\mathrm{B} B \mathrm{BD}$ in the ROls of the tibia in the orthotopic osteosarcoma inhibition experiment. $f H \& E$ staining analysis of lung metastasis in mice in different groups. $g$ TIR of DOX + DDP, DOX + DDP + R848, DDPNPDOX, NPR848, and DDPNPDOX\&R848 in this experiment. Data are presented as the mean $\pm S D(n=8$ for $b-d$ and $g ; n=3$ for $h-i ; * P<0.05$, $* * P<0.01$, $* \star \star P<0.001)$.
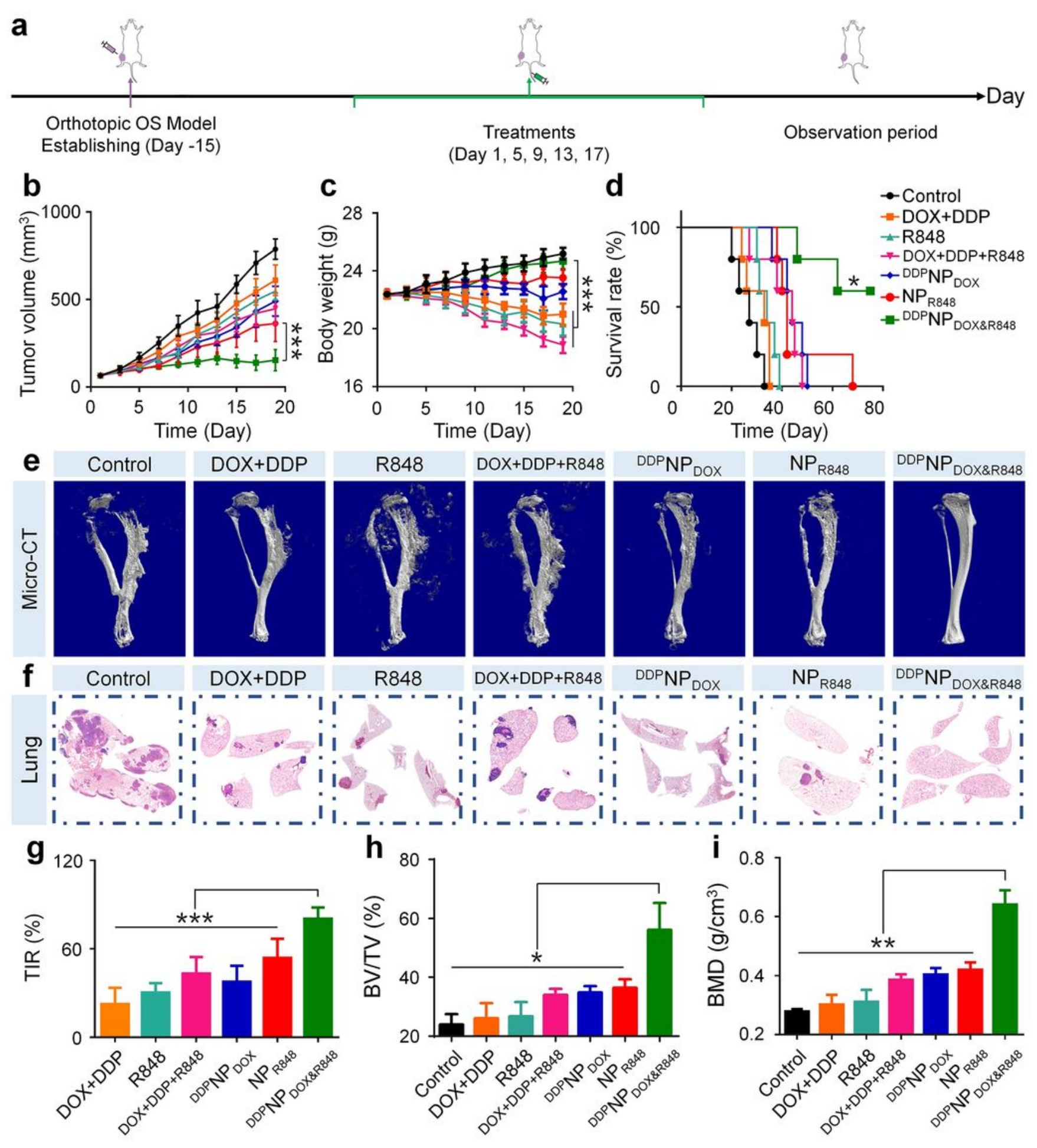

Figure 4 
Antitumor effect in vivo a Experimental schedule for tumor induction and drug treatments. b Tumor volume, $c$ body weight, and d survival rate of mice after various treatments in seven groups. e 3D reconstructed images of the tibia in the orthotopic osteosarcoma inhibition experiment performed using micro-CT and $\mathrm{h}$ BV/TV and $\mathrm{B}$ BMD in the ROls of the tibia in the orthotopic osteosarcoma inhibition experiment. $f$ H\&E staining analysis of lung metastasis in mice in different groups. $g$ TIR of DOX + DDP, DOX + DDP + R848, DDPNPDOX, NPR848, and DDPNPDOX\&R848 in this experiment. Data are presented as the mean $\pm S D(n=8$ for $b-d$ and $g ; n=3$ for $h-i ; * P<0.05$, $* * P<0.01$, $* \star * P<0.001)$.
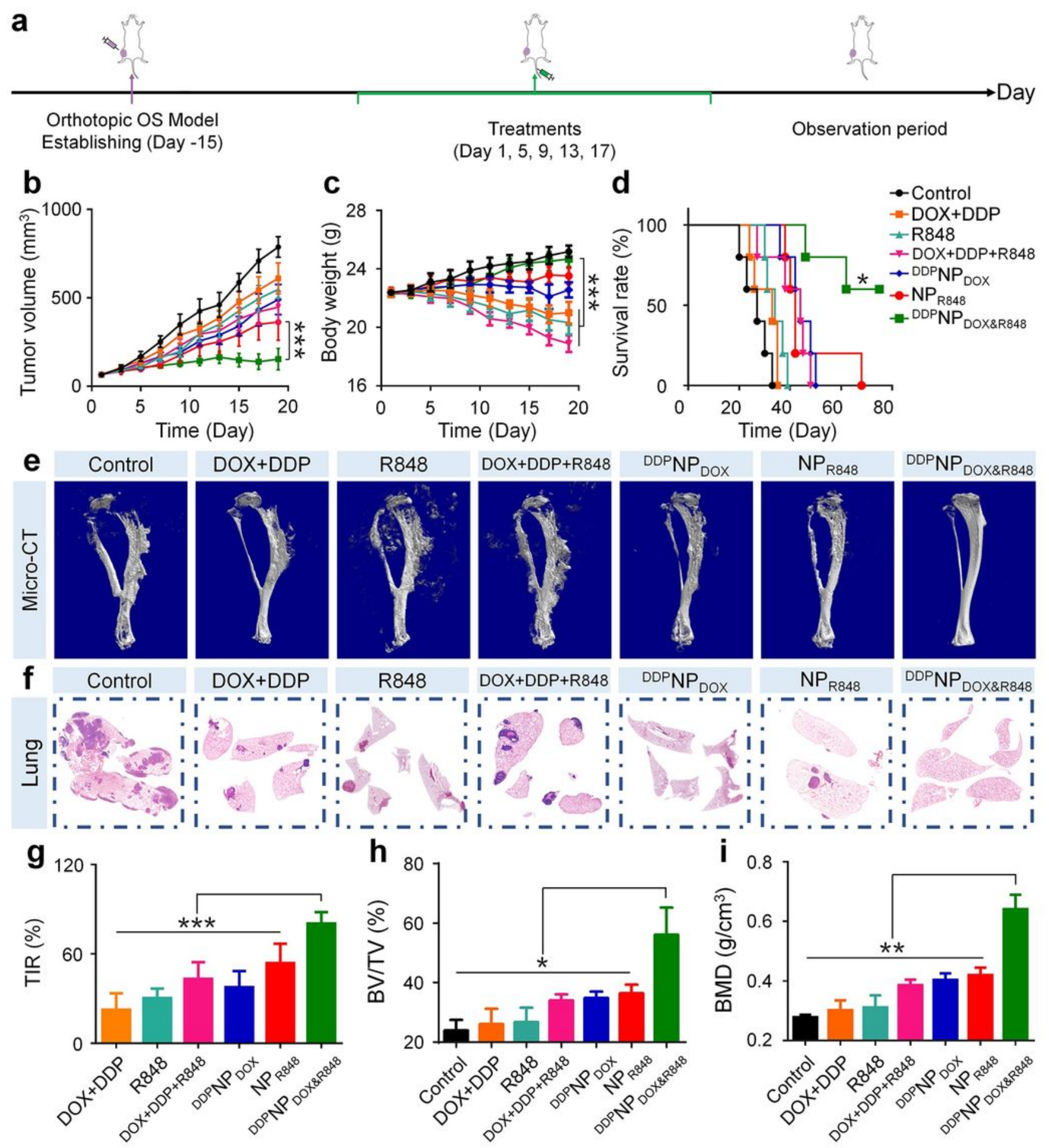


\section{Figure 4}

Antitumor effect in vivo a Experimental schedule for tumor induction and drug treatments. b Tumor volume, $c$ body weight, and d survival rate of mice after various treatments in seven groups. e 3D reconstructed images of the tibia in the orthotopic osteosarcoma inhibition experiment performed using micro-CT and $\mathrm{h}$ BV/TV and $\mathrm{BMD}$ in the ROls of the tibia in the orthotopic osteosarcoma inhibition experiment. $f H \& E$ staining analysis of lung metastasis in mice in different groups. $g$ TIR of DOX + DDP, DOX + DDP + R848, DDPNPDOX, NPR848, and DDPNPDOX\&R848 in this experiment. Data are presented as the mean $\pm S D(n=8$ for $b-d$ and $g ; n=3$ for $h-i ; * P<0.05$, $* * P<0.01, * \star * P<0.001)$. 
a

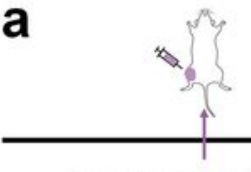

Orthotopic OS Model

Establishing (Day -15)

b

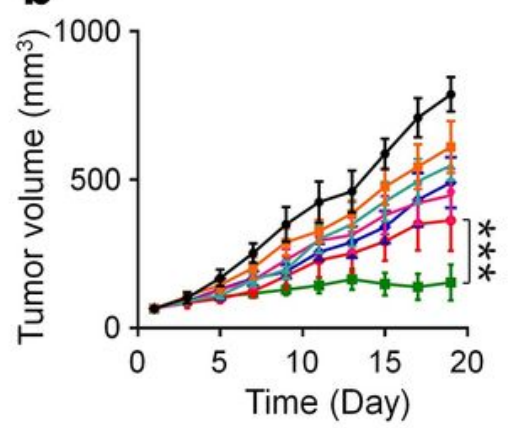

e Control DOX+DDP

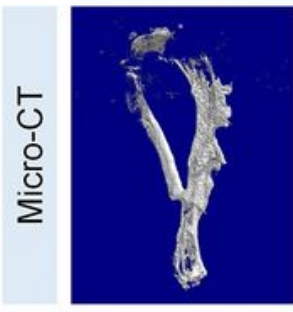

f

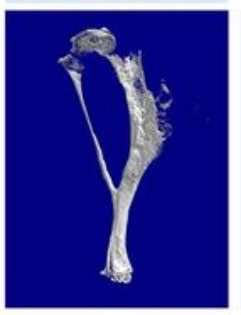

DOX+DDP
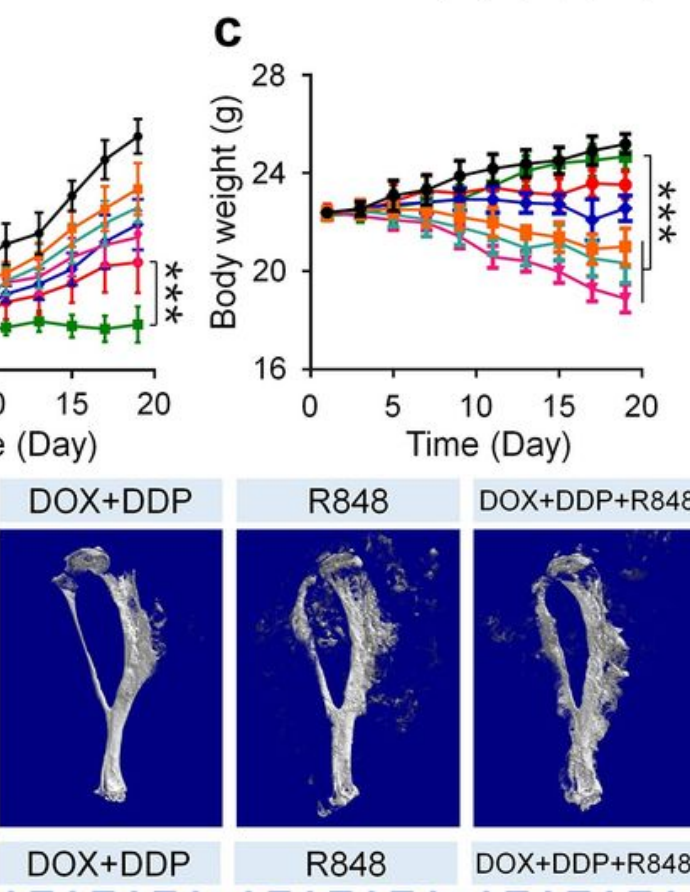

Treatments

(Day 1, 5, 9, 13, 17 )

R848 DOX+DDP+R848
Day

Observation period

d

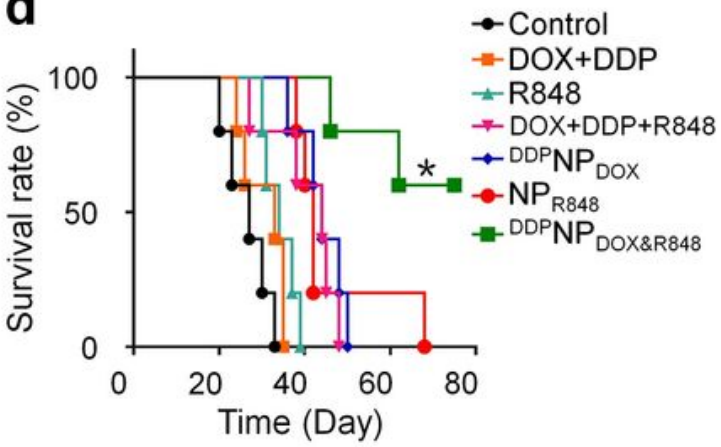

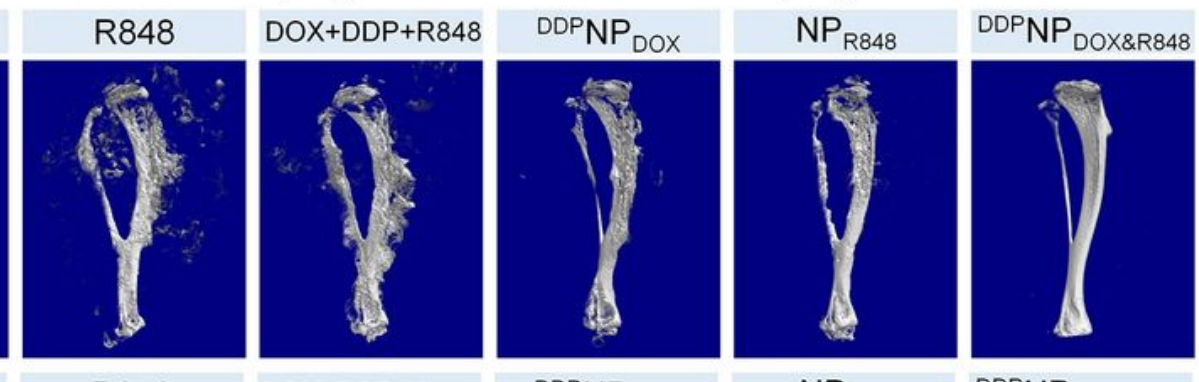

DDPNP

$N P_{\text {R848 }}$

DDPNP DOX\&R848

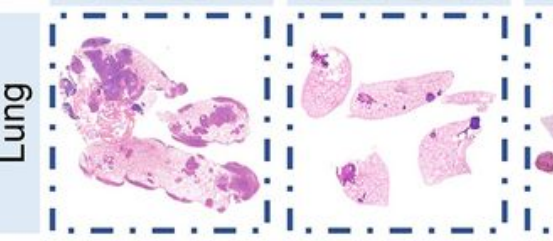

g

120

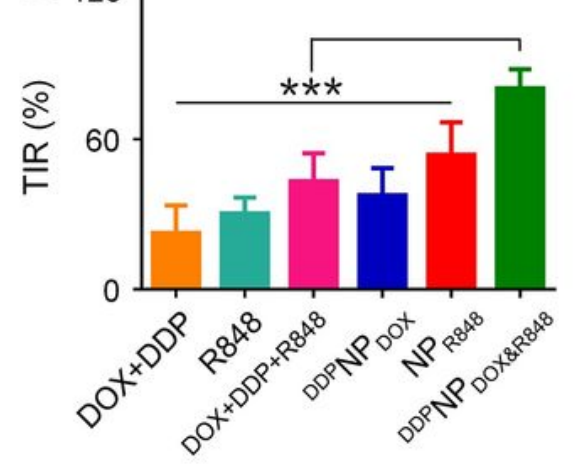

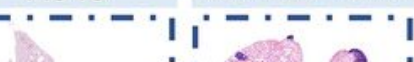
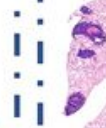
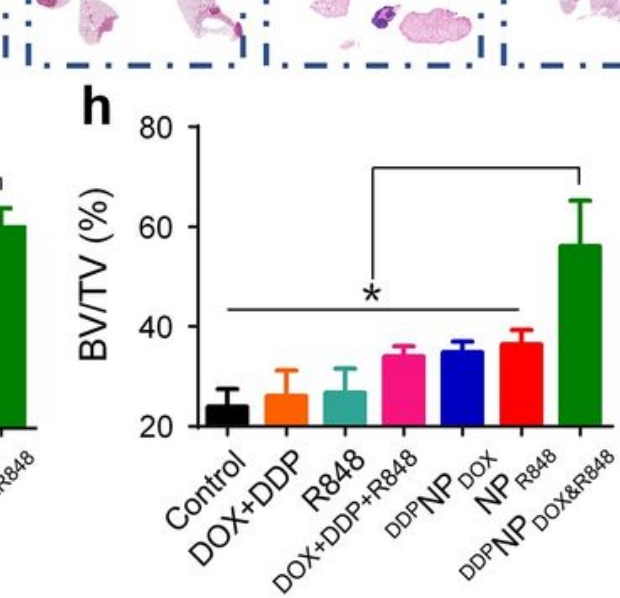
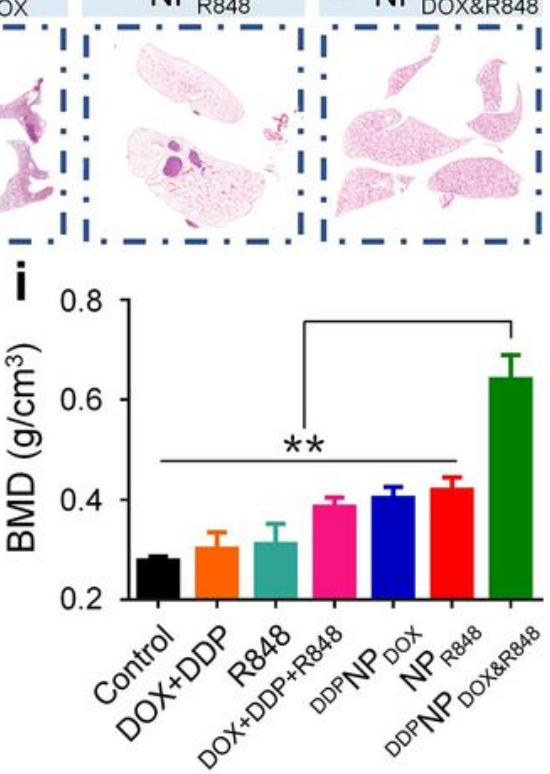

Figure 4

Antitumor effect in vivo a Experimental schedule for tumor induction and drug treatments. b Tumor volume, c body weight, and d survival rate of mice after various treatments in seven groups. e 3D reconstructed images of the tibia in the orthotopic osteosarcoma inhibition experiment performed using micro-CT and $\mathrm{h} \mathrm{BV/TV}$ and $\mathrm{i}$ BMD in the ROls of the tibia in the orthotopic osteosarcoma inhibition experiment. $f$ H\&E staining analysis of lung metastasis in mice in different groups. $g$ TIR of DOX + DDP, 
DOX + DDP + R848, DDPNPDOX, NPR848, and DDPNPDOX\&R848 in this experiment. Data are presented as the mean \pm SD $(n=8$ for $b-d$ and $g ; n=3$ for $h-i ; * P<0.05$, ** $P<0.01$, *** $P<0.001)$.

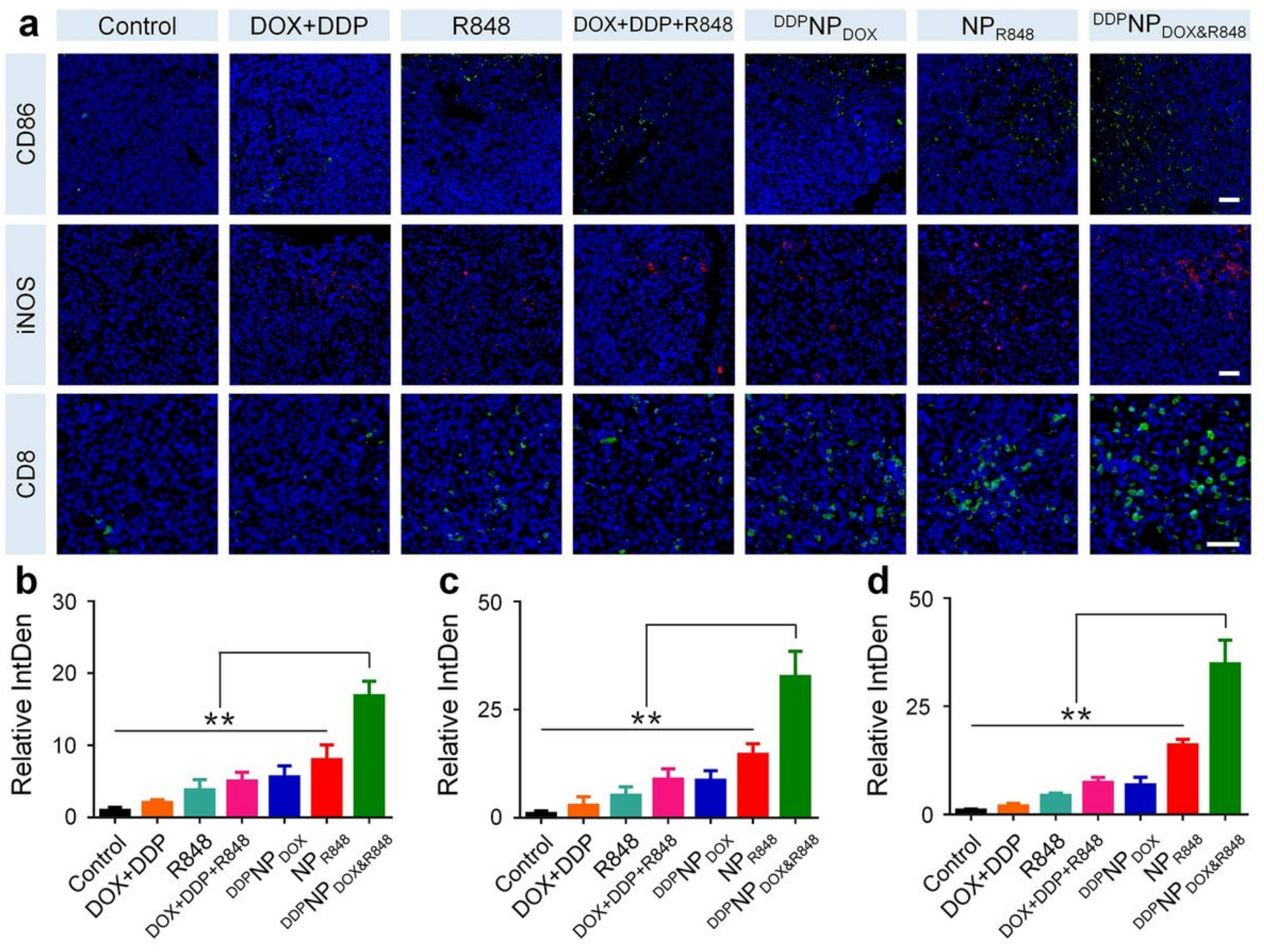

Figure 5

Immune response in vivo a Immunofluorescence (CD86, iNOS, and CD8) analyses and b - $d$ the relative optical densities of tumor tissue sections after different treatments. Scale bars: $50 \mu \mathrm{m}$. Data are presented as the mean $\pm S D(n=3$ for $b-d ; * * P<0.01)$. 


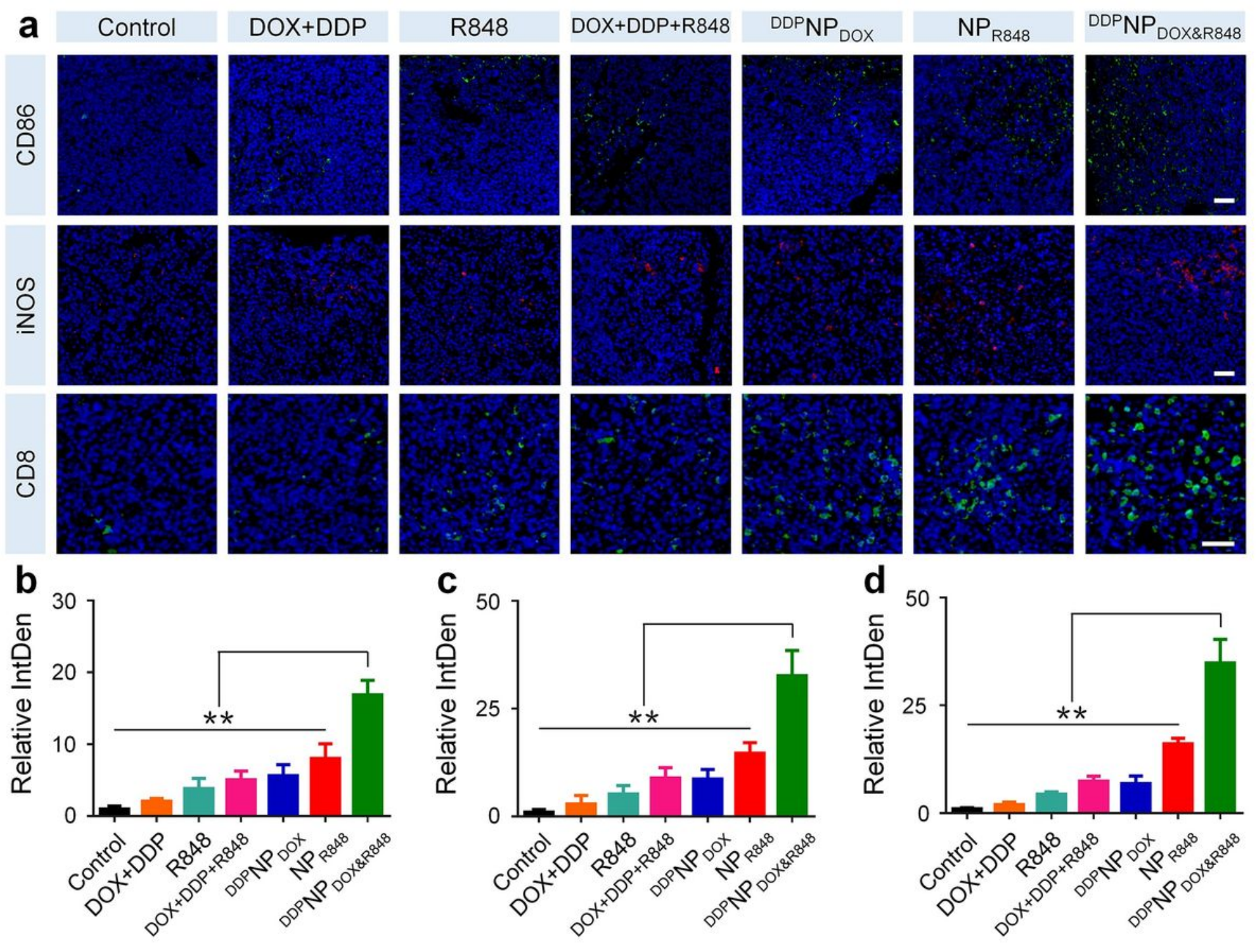

Figure 5

Immune response in vivo a Immunofluorescence (CD86, iNOS, and CD8) analyses and $b-d$ the relative optical densities of tumor tissue sections after different treatments. Scale bars: $50 \mu \mathrm{m}$. Data are presented as the mean $\pm S D(n=3$ for $b-d ; * * P<0.01)$. 


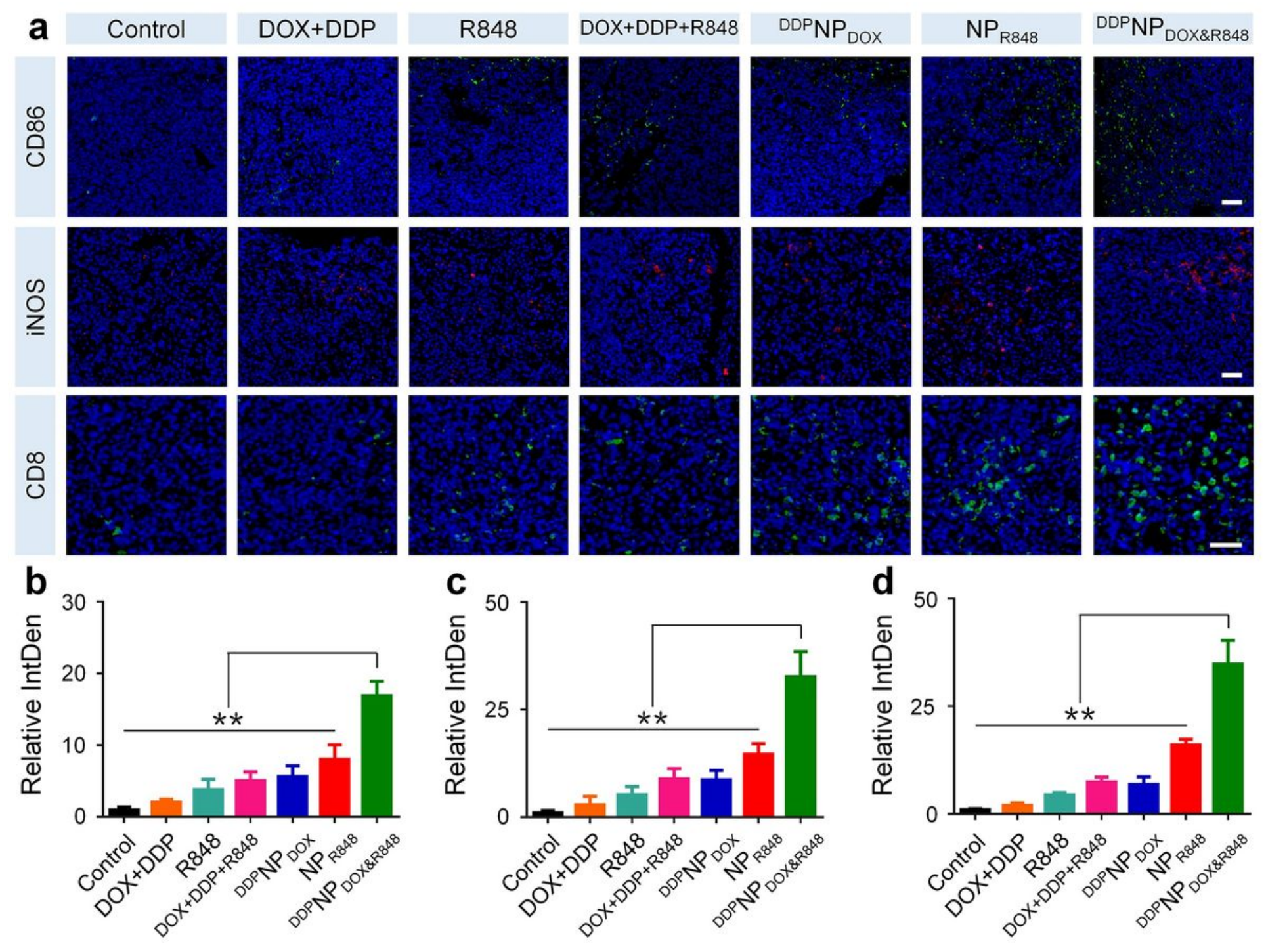

Figure 5

Immune response in vivo a Immunofluorescence (CD86, iNOS, and CD8) analyses and b - $d$ the relative optical densities of tumor tissue sections after different treatments. Scale bars: $50 \mu \mathrm{m}$. Data are presented as the mean $\pm S D(n=3$ for $b-d ; * * P<0.01)$. 


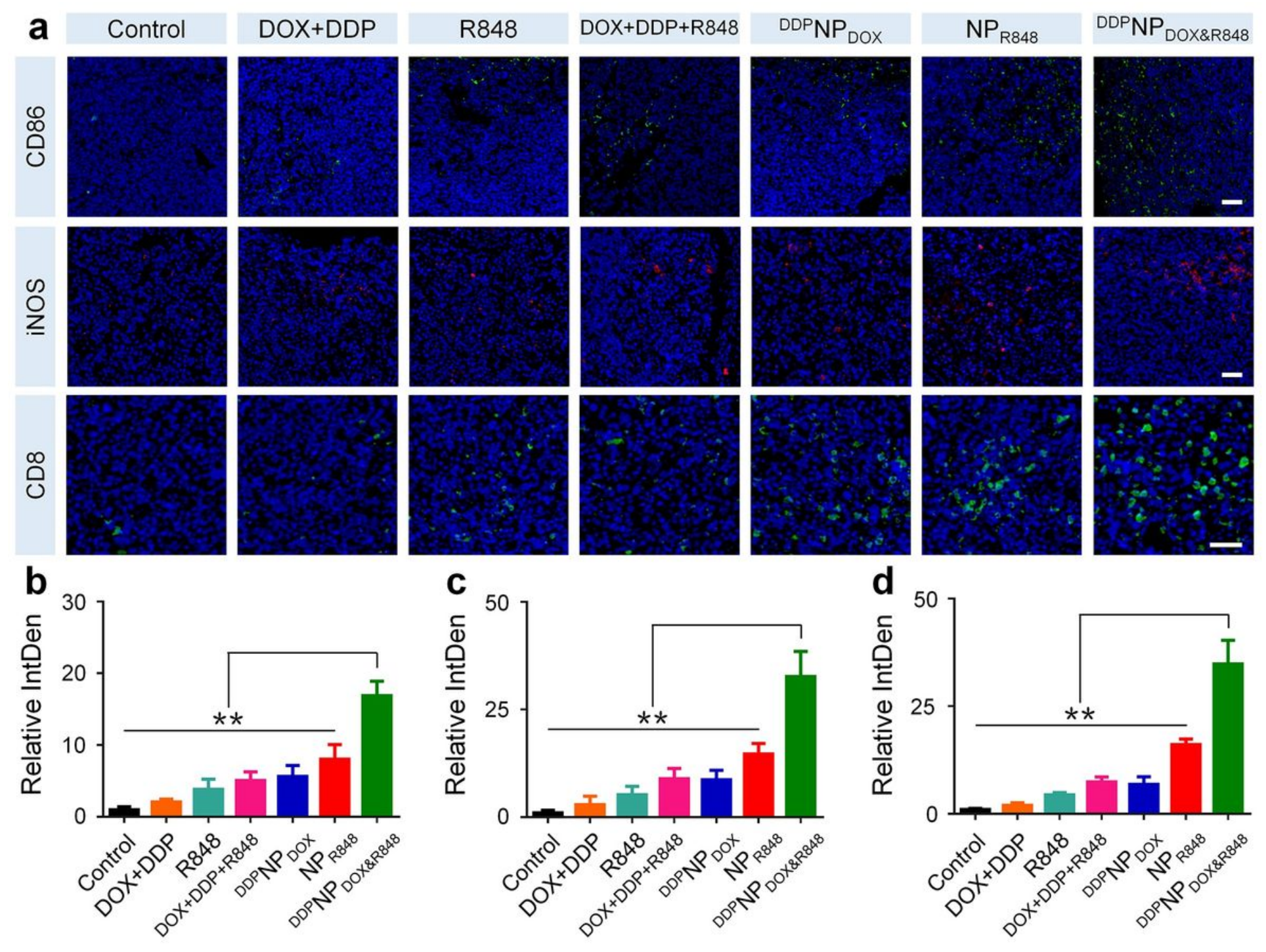

Figure 5

Immune response in vivo a Immunofluorescence (CD86, iNOS, and CD8) analyses and b - $d$ the relative optical densities of tumor tissue sections after different treatments. Scale bars: $50 \mu \mathrm{m}$. Data are presented as the mean $\pm S D(n=3$ for $b-d ; * * P<0.01)$. 


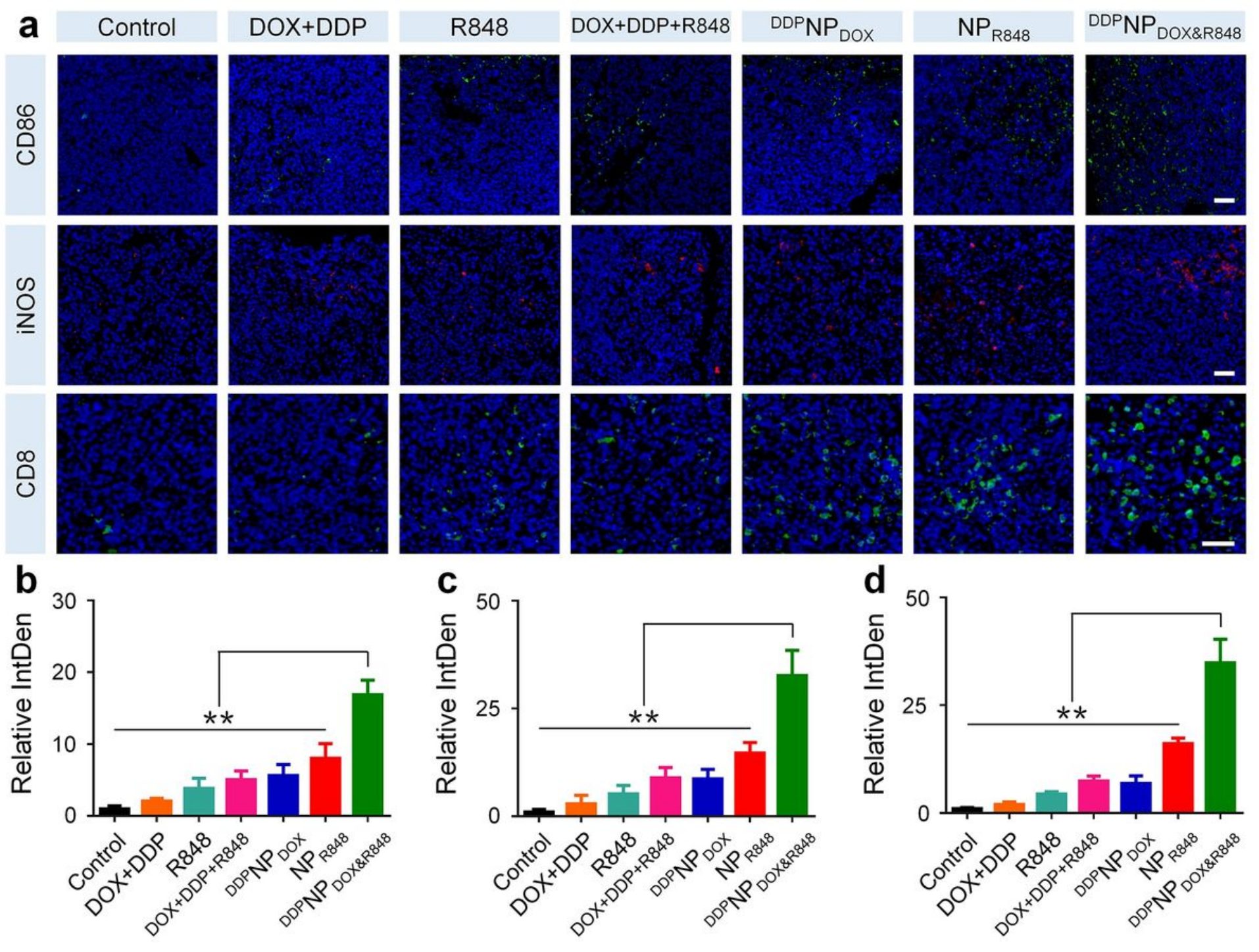

Figure 5

Immune response in vivo a Immunofluorescence (CD86, iNOS, and CD8) analyses and $b-d$ the relative optical densities of tumor tissue sections after different treatments. Scale bars: $50 \mu \mathrm{m}$. Data are presented as the mean $\pm S D(n=3$ for $b-d ; * * P<0.01)$. 


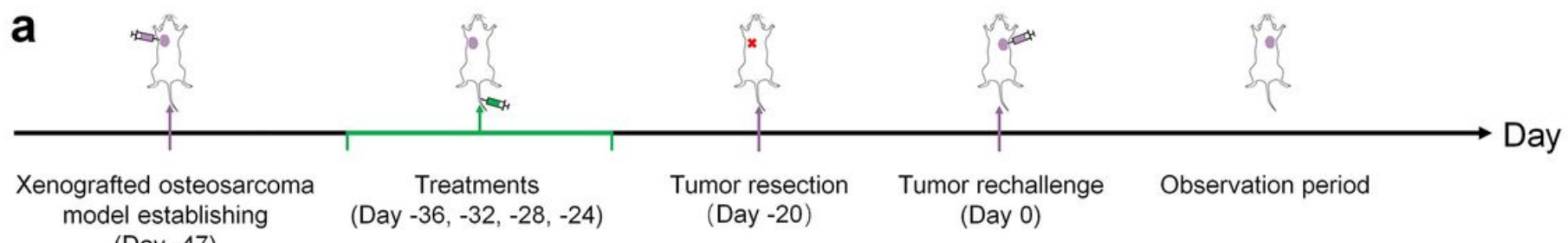
(Day -47)
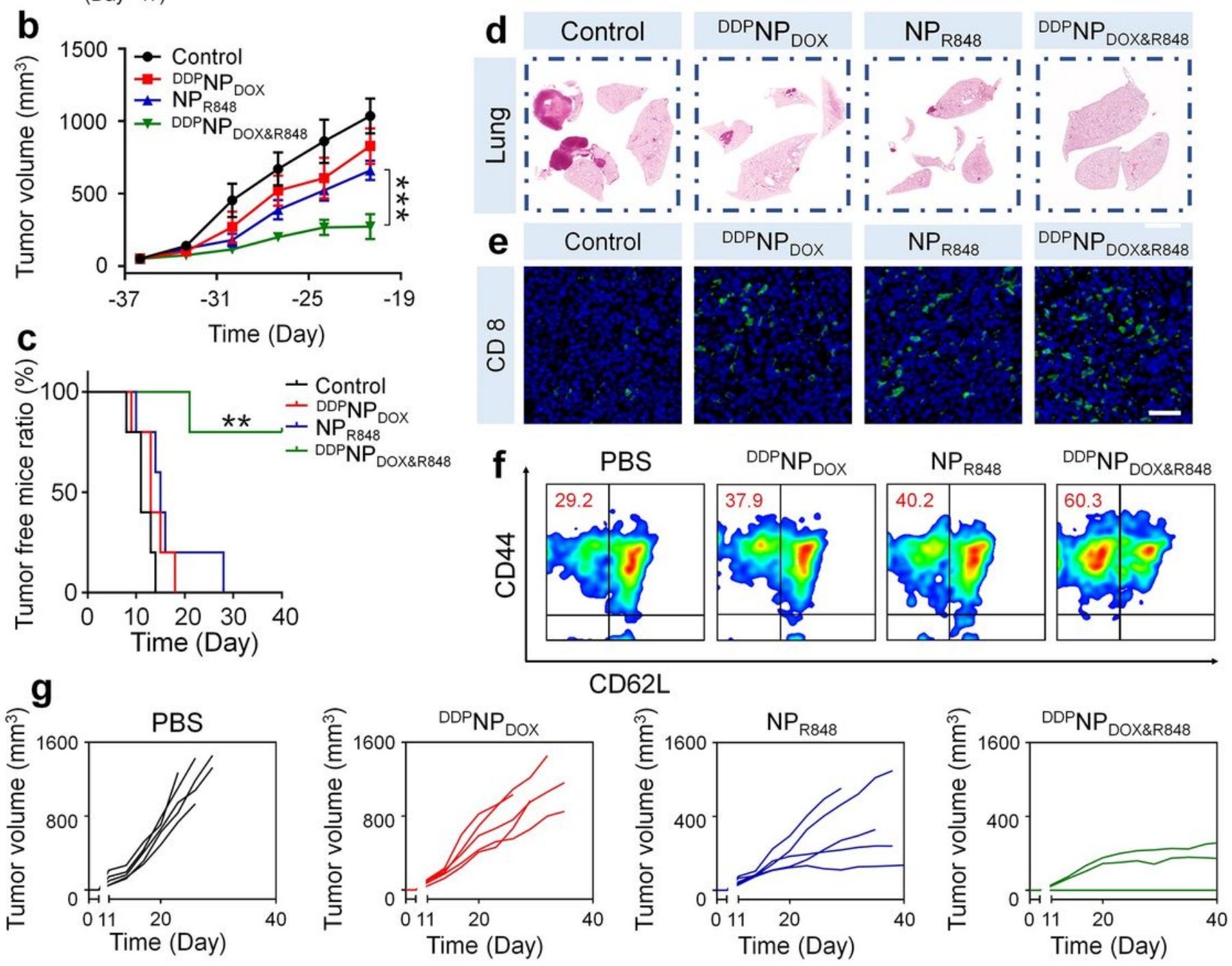

\section{Figure 6}

Long-term immune memory effect a Experimental schedule. $\mathrm{b}$ The primary tumor volume and c tumor free mice ratio of mice treated with PBS, DDPNPDOX, NPR848, and DDPNPDOX\&R848. $d$ H\&E staining analysis of lung metastasis in mice in different groups. e l3unofluorescence (CD8) analyses of tumor tissue sections after different treatments. Scale bars: $50 \mu \mathrm{m}$. $f$ The percent of TEM cells in spleens of the mice with different treatments were analyzed by flow cytometry (gated on CD3+ CD8+ T cells). g Individual tumor growth kinetics of the rechallenged distant tumors in mice with different treatments. Data are presented as the mean $\pm S D\left(n=8\right.$ for $b$ and $n=5$ for $c$; $\left.{ }^{*} P<0.01\right)$. 


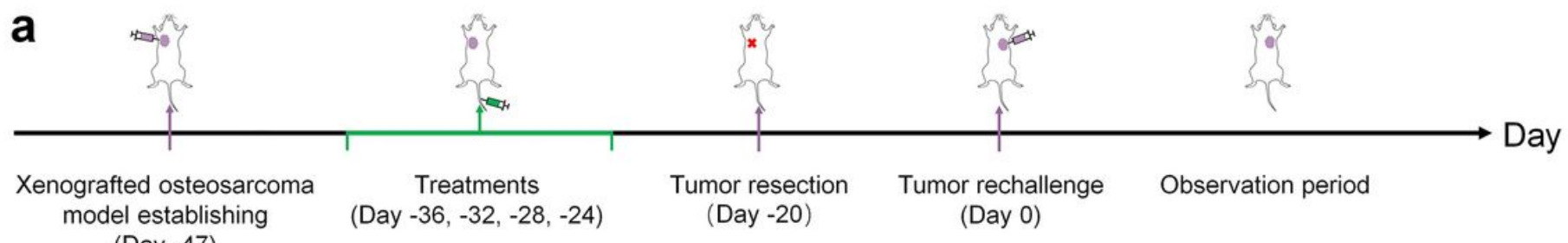
(Day -47)
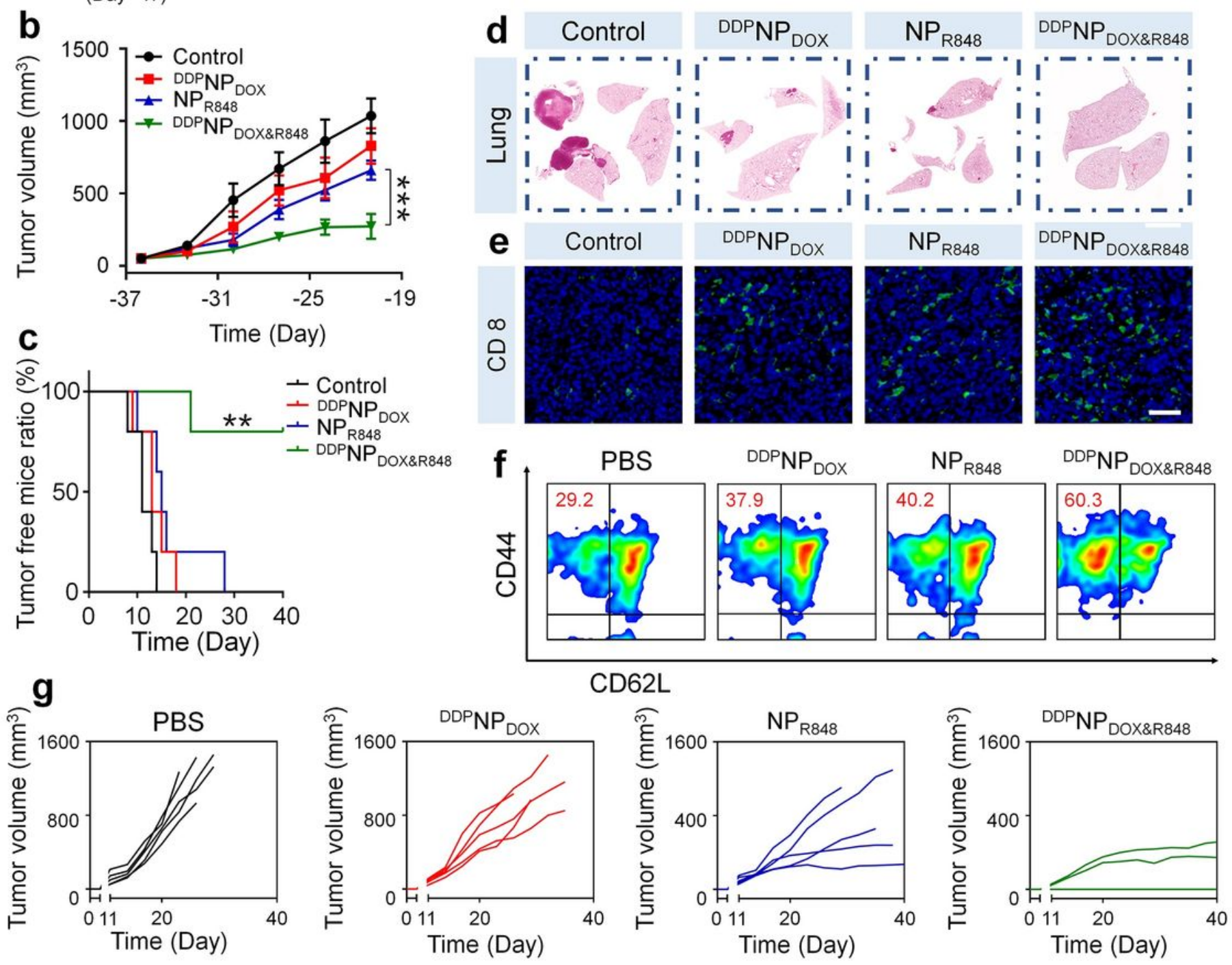

\section{Figure 6}

Long-term immune memory effect a Experimental schedule. $\mathrm{b}$ The primary tumor volume and c tumor free mice ratio of mice treated with PBS, DDPNPDOX, NPR848, and DDPNPDOX\&R848. $d$ H\&E staining analysis of lung metastasis in mice in different groups. e l3unofluorescence (CD8) analyses of tumor tissue sections after different treatments. Scale bars: $50 \mu \mathrm{m}$. $f$ The percent of TEM cells in spleens of the mice with different treatments were analyzed by flow cytometry (gated on CD3+ CD8+ T cells). g Individual tumor growth kinetics of the rechallenged distant tumors in mice with different treatments. Data are presented as the mean $\pm S D\left(n=8\right.$ for $b$ and $n=5$ for $c$; $\left.{ }^{*} P<0.01\right)$. 


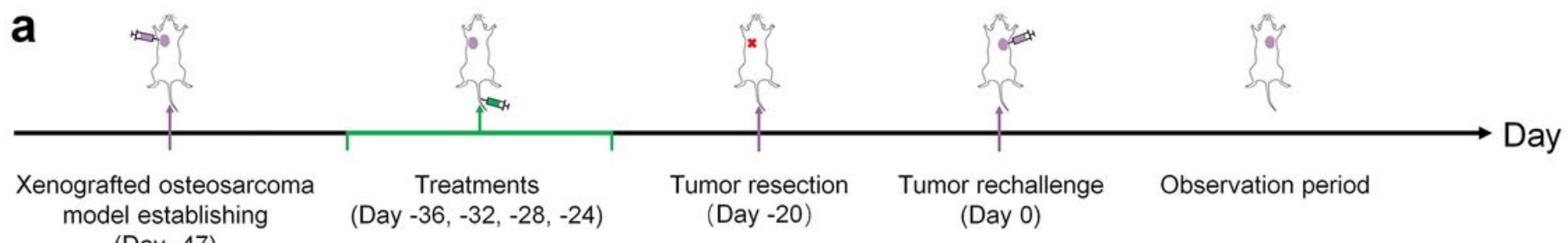
(Day -47)
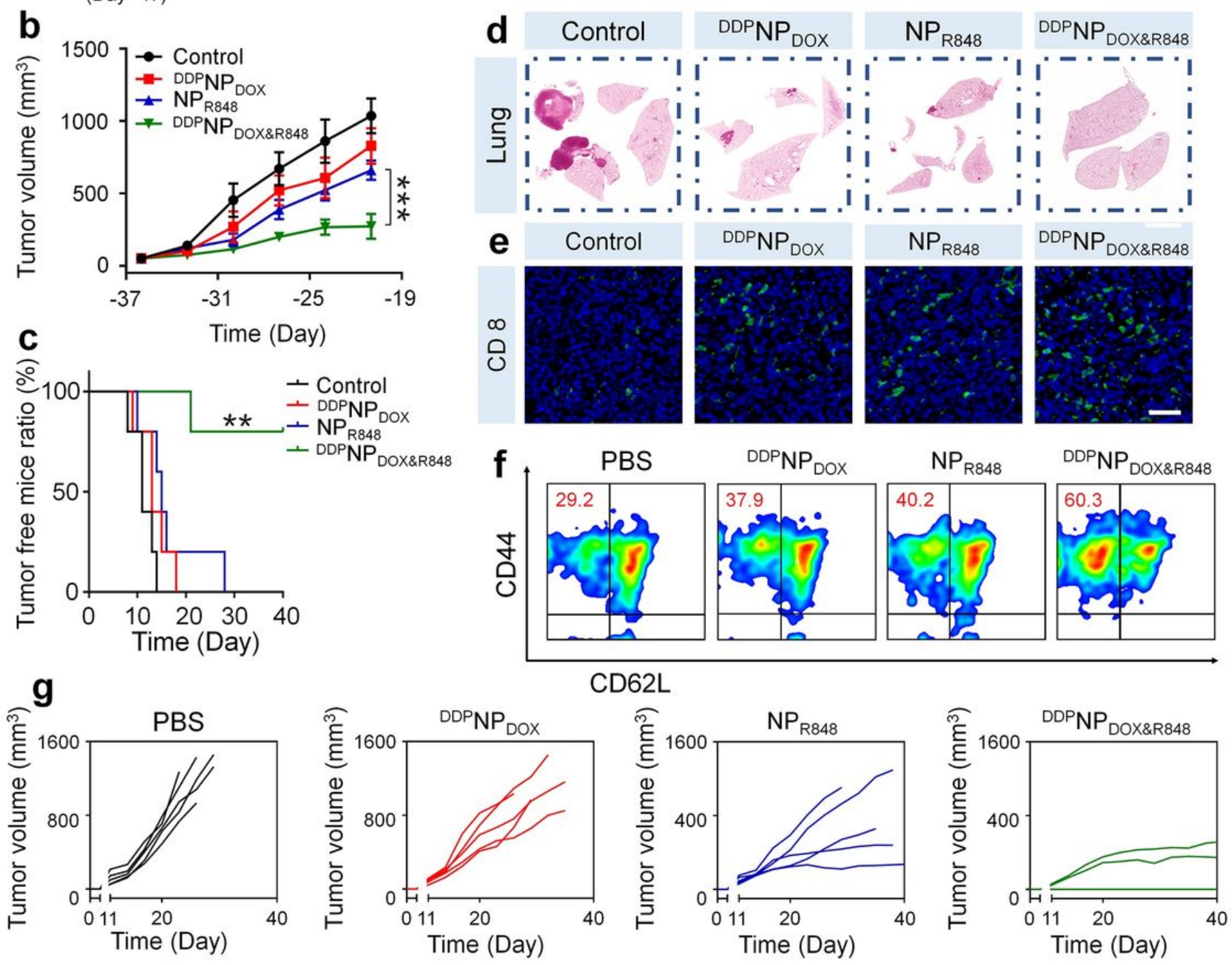

\section{Figure 6}

Long-term immune memory effect a Experimental schedule. $\mathrm{b}$ The primary tumor volume and c tumor free mice ratio of mice treated with PBS, DDPNPDOX, NPR848, and DDPNPDOX\&R848. $d$ H\&E staining analysis of lung metastasis in mice in different groups. e l3unofluorescence (CD8) analyses of tumor tissue sections after different treatments. Scale bars: $50 \mu \mathrm{m}$. $f$ The percent of TEM cells in spleens of the mice with different treatments were analyzed by flow cytometry (gated on CD3+ CD8+ T cells). g Individual tumor growth kinetics of the rechallenged distant tumors in mice with different treatments. Data are presented as the mean $\pm S D\left(n=8\right.$ for $b$ and $n=5$ for $c$; $\left.{ }^{*} P<0.01\right)$. 


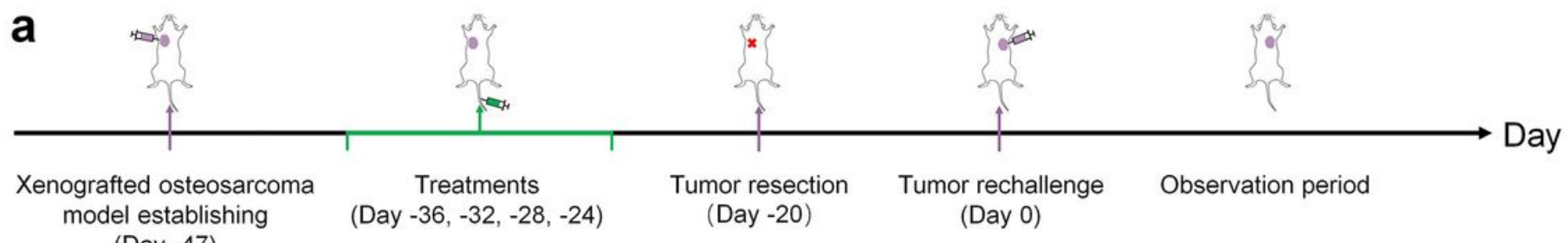
(Day -47)
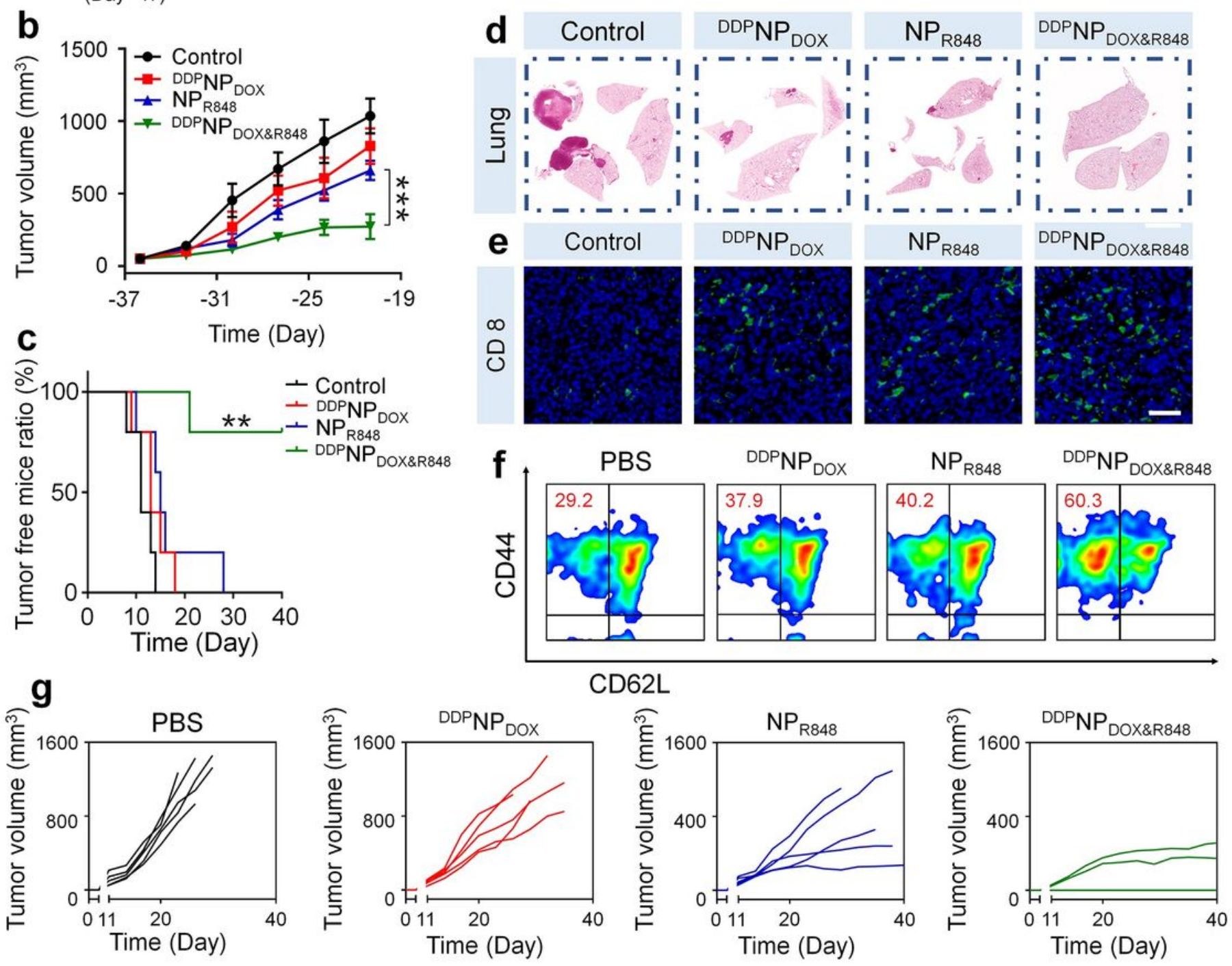

\section{Figure 6}

Long-term immune memory effect a Experimental schedule. $\mathrm{b}$ The primary tumor volume and c tumor free mice ratio of mice treated with PBS, DDPNPDOX, NPR848, and DDPNPDOX\&R848. $d$ H\&E staining analysis of lung metastasis in mice in different groups. e l3unofluorescence (CD8) analyses of tumor tissue sections after different treatments. Scale bars: $50 \mu \mathrm{m}$. $f$ The percent of TEM cells in spleens of the mice with different treatments were analyzed by flow cytometry (gated on CD3+ CD8+ T cells). g Individual tumor growth kinetics of the rechallenged distant tumors in mice with different treatments. Data are presented as the mean $\pm S D\left(n=8\right.$ for $b$ and $n=5$ for $c$; $\left.{ }^{*} P<0.01\right)$. 


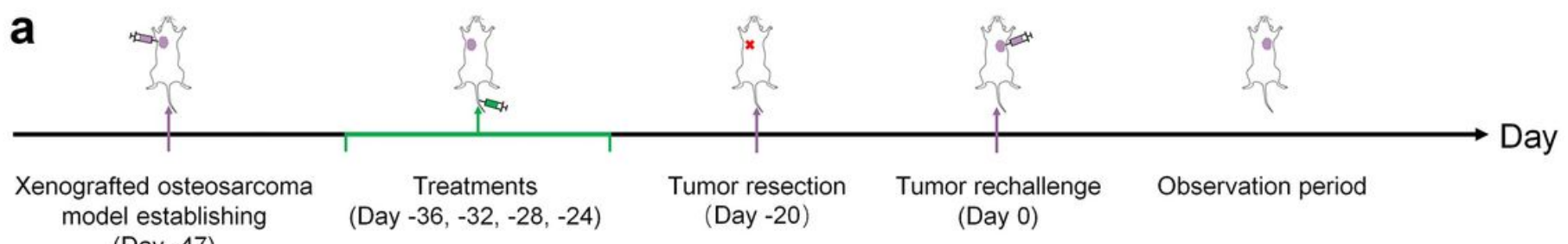
(Day -47)
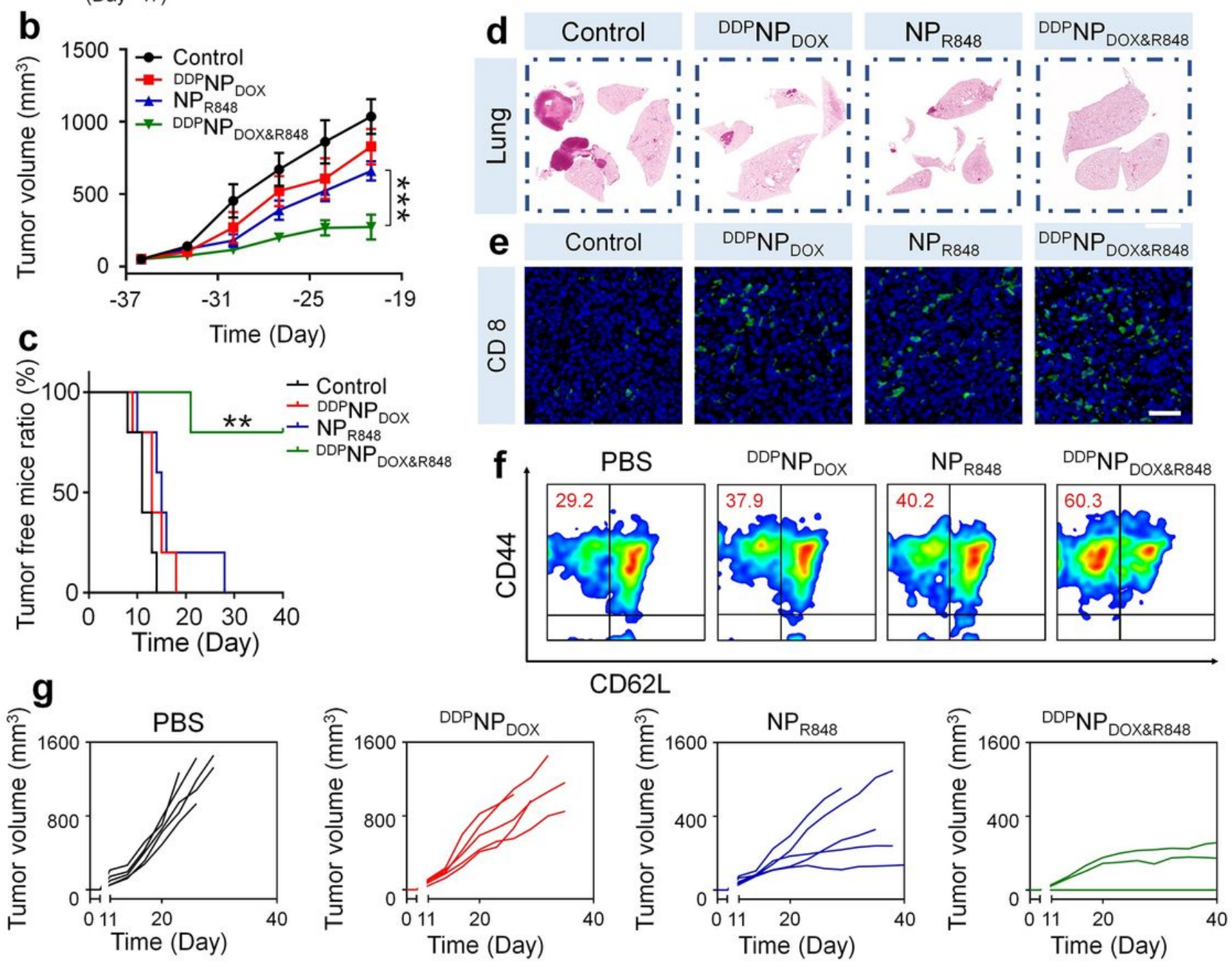

\section{Figure 6}

Long-term immune memory effect a Experimental schedule. $\mathrm{b}$ The primary tumor volume and c tumor free mice ratio of mice treated with PBS, DDPNPDOX, NPR848, and DDPNPDOX\&R848. $d$ H\&E staining analysis of lung metastasis in mice in different groups. e l3unofluorescence (CD8) analyses of tumor tissue sections after different treatments. Scale bars: $50 \mu \mathrm{m}$. $f$ The percent of TEM cells in spleens of the mice with different treatments were analyzed by flow cytometry (gated on CD3+ CD8+ T cells). g Individual tumor growth kinetics of the rechallenged distant tumors in mice with different treatments. Data are presented as the mean $\pm S D\left(n=8\right.$ for $b$ and $n=5$ for $c$; $\left.{ }^{*} P<0.01\right)$. 